\title{
Non Unitary Random Walks
}

\author{
Philippe Jacquet
}

INRIA, Le Chesnay, France

received July 30, 2009, revised Apr 19, 2010, accepted May 5, 2010.

\begin{abstract}
Motivated by the recent refutation of information loss paradox in black hole by Hawking, we investigate the new concept of non unitary random walks. In a non unitary random walk, we consider that the state $s_{0}$, called the black hole, has a probability weight that decays exponentially in $e^{-\lambda t}$ for some $\lambda>0$. This decaying probabilities affect the probability weight of the other states, so that the the apparent transition probabilities are affected by a repulsion factor that depends on the factors $\lambda$ and black hole lifetime $t$. If $\lambda$ is large enough, then the resulting transition probabilities correspond to a neutral random walk. We generalize to non unitary gravitational walks where the transition probabilities are function of the distance to the black hole. We show the surprising result that the black hole remains attractive below a certain distance and becomes repulsive with an exactly reversed random walk beyond this distance. This effect has interesting analogy with the so-called dark energy effect in astrophysics.
\end{abstract}

Keywords: random walks, quantum systems, analysis of algorithms, generating function, continuous fractions, singularity analysis

\section{Introduction and motivation}

A black hole is a massive stellar object that absorbs everything that get trapped in its gravitational field, including light. In 1976, the famous astrophysicist Stephen Hawking [2], predicted that the black holes cannot be absolutely black and eternal. Indeed black holes evaporate via Hawking radiation and eventually disappear. A major problem was detected in the fact that Hawking radiation should not carry any information and therefore the informations contained in the objects absorbed by the black hole during its lifetime simply disappear. This information loss paradox leads to fundamental question in theoretical physics since it apparently violates the principle of unitarity in quantum physics (more details are given in our Section 22). There are many studies related to the question of non unitary quantum gravity. Some of these studies has given the opportunity of numerical simulations of non trivial complex systems [8].

In 2006 Hawking [11] refuted his original argument about the information loss paradox. His refutation is still an object of controversy but established the foundation of non unitary Markov systems. Basically Hawking stated in his 2006 paper that if black holes would host a superposition of unitary and non unitary metrics, then the the contributions of non unitary metric would vanish exponentially with time, and therefore only the effect in unitary metric would remain at infinity. In other words a black hole tends almost surely to be unitary and thus to release its information without loss during its lifetime.

The motivation of this paper is to help in some of the computational aspects in the analysis of non unitary Markov systems. We describe a simple model of non unitary effects where some probability 
weights decay exponentially with time. What is interesting is the effect of the unitary loss on a halo of matter that surrounds a black hole. The matter acts like a gas where particles collide at random times like in a random walk. In physics, random walk problems are investigated via finite element simulations where particles travel between finite boxes of space. Each box is a state in the random walk. In a non unitary random walk, we consider that the state $s_{0}$, called the black hole state, is an absorbing state and has a probability weight that decays exponentially in $e^{-\lambda t}$ for some $\lambda>0$.

We will show that such non unitary random walks show a behavior which is very sensitive to their tuning parameters, and thus the simulation must be done in the most realistic situation. In this case as in most simulation of large systems in astrophysics we hit some computational boundaries even when using the most powerful super-computers [10]. Indeed, the number of states in a realistic random walk in a galactic halo may exceed $10^{20}$, even after merging a majority of states due to the spherical symmetry. This makes the simulation very hard to process. Furthermore, as we will show later, the impact of the unitarity effect stands in the future of the particle, namely in the quantity of probability weight it will lose after eventually being ingested by the black hole. Therefore the simulations must compute the probability weight of all potential trajectories in the future. In a (classic) unitary universe, all these trajectories sums to one. But in a non unitary universe this is not the case, and computation must involve all possible trajectories, at least within the whole life time of the black hole. A super-massive black hole, like the black hole which lies in the center of our galaxy, has a lifetime of order $10^{90}$ years. Such simulation is rather impossible to make: either we have to iterate on a vector of $10^{20}$ coefficients over $10^{90}$ steps, or we iterate during $\log _{2} 10^{90}$ steps, on the successive squares of the non unitary matrix, which is of size $10^{20} \times 10^{20}$.

The object of this paper is to analytically guess the results of such simulations by investigating the asymptotics of non unitary random walks. We show the surprising result that the black hole remains attractive below a certain distance and becomes repulsive or at least neutral beyond this distance. This effect shows an interesting analogy with so-called dark energy effect in astrophysics.

One should not confuse the methodology introduced in this paper with the beautiful analyses in [6, 12] where the particular nature of the quantum random walk is contained in the state superposition in the wave function. Here we analysis the random walks in a more classic way without state superposition, the quantum non unitary effect being contained in the unique black hole absorbing state of the random walk. We base our analysis on the framework of many previous fundamental works, i.e. the representation of generating functions of random walks as continuous fractions in [1], the enumeration of paths in random walks in [7], the singularity analysis in the generating functions in [3]. Our work comes in parallel of related works on the use of combinatorics and generating functions on random walks [4, 9],

The paper is organized as follow.

- In the two next Sections 2 and 3 we describe non unitary effects and analyse a simple model of non unitary Markov system, with only two states.

- In Section 3, we extend the analysis over the non unitary Markov systems that contains an infinite number of states, so-called non unitary random walks. We first investigate the impact of non unitary effect on uniform random walks.

- In Section 4 we investigate the case of non uniform random walks, so-called concave random walks. To this end we make use of continued fraction generating function representation and show asymptotic results about their behavior around main singularities. We show that under some circonstance, 
the non unitarity effect of the black hole makes the random walk apparently bimodal, being apparently attractive below a certain state and repulsive beyond that state.

- We finish our paper on the special case of gravitational walks (Section 5) and briefly discuss some physical considerations (Section 6), that is the consequence of the bimodal behavior we have found.

\section{Non Unitary effect and the Schrödinger's rabbit}

In a classic unitary universe all probabilities sum to one. If you take a rabbit at time $t=0$ then one month later the rabbit will be either alive with probability $\alpha>0$ or dead with probability $\beta>0$ and $\alpha+\beta=1$. But in quantum physics the sum of all probabilities is interpreted as being the integral $\int|\Psi|^{2}$ of the wave function $\Psi$ of the universe. Therefore the sum of probabilities being equal to one is no more a mathematical statement but a physical statement. Unitarity principle states that for all time $t: \int|\Psi|^{2}=1$.

But in a non unitary universe we may have $\int|\Psi|^{2} \neq 1$. The loss of information implies a non unitary universe. Hawking describes metrics pertinent for black holes (so-called anti-de-Sitter metrics) where $\int|\Psi|^{2}=e^{-\lambda t}$ for some $\lambda>0$. In other words let assume a rabbit which at time $t=0$ is either (with probability $\alpha$ in state $s_{0}$ inside a black hole embedded with a non unitary metric, or (with probability $\beta$ ) in state $s_{1}$ outside a black hole in an unitary metric. If the black hole lifetime is $t$ then at time $t$ the probability sum of state $s_{0}$ will be $r_{0}(t)=e^{-\lambda t}$ while the probability sum of state $s_{1}$ will remain at $r_{1}(t)=1$. Consequently the sum of probabilities of the rabbit equals $\alpha r_{0}(t)+\beta r_{1}(t) \neq 1$ and when $t$ increases the apparent probability of state $s_{0}$ defined as $\frac{\alpha r_{0}(t)}{\alpha r_{0}(t)+\beta r_{1}(t)}$ tends to 0 . In other words the rabbit never falls in the black hole and stays outside.

This is equivalent in finance, to saying that an investor with one euro at time $t=0$ puts $\alpha$ cents in a black hole market, and keeps $\beta$ cents in his pocket. If the black hole market looses value at rate $-\lambda$, then at the end of day the investor will have most of his remaining fortune in his pocket.

In fact Hawking's point is not as trivial as exposed above: he does not consider a rabbit, but the whole black hole and considers that its quantum state is a superposition of unitary and non unitary metrics. He shows that on the path integral over the black hole lifetime, the contributions of non unitary metric vanish exponentially, so that only the unitary metric contributions remain.

In the following we are not considering state superposition, we assume that either the rabbit is inside the black hole (state $s_{0}$ ) or far away outside the black hole (state $s_{1}$ ). This hypothesis is realistic since the two possible space locations of the rabbit are so far apart that quantum state overlaps are negligible. Therefore we treat the problem in a classical way. We can see the rabbit as a classical equivalent of Schrödinger cat in an non unitary universe. The original Schrödinger cat can be in the superposition of two states $s_{0}$ and $s_{1}$ (for instance dead or alive). We introduce the Schrödinger rabbit which is either in state $s_{0}$ or in state $s_{1}$, but the probability sum of state $s_{0}$ decays exponentially with time.

\section{Modelling non unitary systems}

\subsection{Non unitary Markov processes}

We still consider a two state process with a state $s_{0}$ and a state $s_{1}$ (see Figure 1). The state $s_{0}$ is an absorbing state that mimics a black hole $(\mathrm{BH})$ and during the black hole lifetime the probability sum decays with rate $-\lambda$. We consider that the time is discretized and during the black hole lifetime, at each time unit the rabbit has a probability $q$ to fall into the black hole, and therefore a probability $p$ to remain 


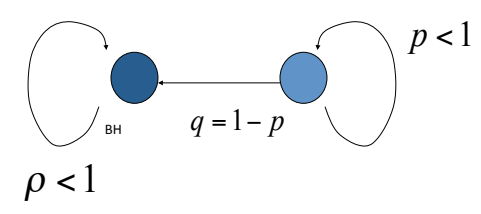

Fig. 1: A two state non unitary system.

outside the black hole during this time slot. When the rabbit falls in the black hole, it stays inside the black hole until the end of the black hole lifetime.

Or in other words, an investor has one euro in its pocket at time $t=0$ and he has a contract with its black hole bank to move every month a fraction $q$ of his pocket money in the black hole market. We call $q$ the attraction probability, and $p$ the repulsion probability.

This system mimics the effect of a black hole which attracts any object in its vicinity. We will see that if the black hole exerts an attraction which is less powerful than its probability weight decay rate, then the black hole is apparently repulsive. Otherwise, if the attraction is more powerful than the probability decay rate, then the result is reversed.

The probability sum of state $s_{0}$ for a black hole remaining lifetime $t$ satisfies $r_{0}(t)=\rho^{t}$. But, about $r_{1}(t)$, the probability sum of state $s_{1}$, the transition from lifetime $t$ and lifetime $t-1$ gives the identity $r_{1}(t)=q r_{0}(t-1)+p r_{1}(t-1)$. This makes also $r_{1}(t)<1$. Notice that the previous identity is time forward, since the black hole lifetime decreases when the time goes forward. We call such system a non unitary Markov process with transition matrix R:

$$
\mathbf{R}=\left[\begin{array}{ll}
\rho & q \\
0 & p
\end{array}\right]
$$

We call the quantity denoted by $\rho$ the probability decay factor. The matrix $\mathbf{R}$ is not unitary in the Markovian sense, since vector $[1,1]$ is not an eigenvector and 1 is not an eigenvalue as soon as $\rho \neq 1$ :

$$
[1,1] \mathbf{R} \neq[1,1] .
$$

The eigenvalues of matrix $\mathbf{R}$ are $\rho$ and $p$. We expect to have a different behavior depending on $p>\rho$ or on $\rho>p$. The objective is to estimate the apparent attraction of the black hole, or more precisely, the apparent repulsion defined below.

We call the quantity $\tilde{p}(t)=p \frac{r_{1}(t-1)}{r_{1}(t)}$, the apparent repulsion probability, and $\tilde{q}(t)=1-\tilde{p}(t)=$ $q \frac{r_{0}(t-1)}{r_{1}(t)}$, the apparent attraction probability. The apparent attraction is equal to the attraction probability multiplied by a factor that takes into account the probability decay that the rabbit will experience inside the black hole until the end of the black hole lifetime $t-1$, if it falls inside the black hole during this time slot. Starting from the final probability weight $\left[r_{0}(0), r_{1}(0)\right]=[1,1]$ (the black hole system returns to unitarity at the end of black hole lifetie), we have

$$
\left[r_{0}(t), r_{1}(t)\right]=[1,1] \mathbf{R}^{t} .
$$

We prove the following very simple theorem whose main purpose is to introduce our methodology 


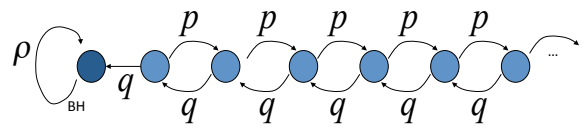

Fig. 2: Non unitary uniform random walk.

Theorem 1 We consider a two-state non unitary Markov model of black hole with state $s_{0}$ and state $s_{1}$. The state $s_{0}$ is the absorbing black hole state and quantity $\rho>0$ is the probability decay factor on the black hole state. The integer $t$ is the remaining black hole lifetime. Quantity $p$ is the repulsion probability defined as the probability that the rabbit stays on state $s_{1}$ during an unit step time. When the probability decay factor is greater than the repulsion probability $p$, then the black hole is apparently attractive when $t \rightarrow \infty$, otherwise when $\rho \leq p$, the black hole is apparently repulsive when $t \rightarrow \infty$.

Proof: We consider the case where $\rho \neq p$. From the fact that the probability sum of state $s_{1}$ satisfies the identity $r_{1}(t)=q \frac{\rho^{t}-p^{t}}{\rho-p}+p^{t}$, we get the apparent repulsion probability $\tilde{p}(t)=\frac{p / r-(p / r)^{t}}{1-(p / r)^{t}}=\frac{p}{\rho}+O\left(\left(\frac{p}{\rho}\right)^{t}\right)$ when $\rho>p$. In this case the attraction is stronger than the non unitary effect and the rabbit eventually falls in the black hole which exerts an apparent attraction probability $\tilde{q}(t)=1-\frac{p}{\rho}+o(t)$ which remains away from zero when $t \rightarrow \infty$. In other words the apparent probability that the rabbit will eventually fall in the black hole which is equal to $1-\prod_{k=0}^{t} \tilde{p}(k)$, tends to 1 when $t \rightarrow \infty$.

In the opposite case, when $\rho<p$, we have $\tilde{p}(t)=1+O\left(\left(\frac{\rho}{p}\right)^{t}\right)$, the non unitary effect is stronger than the attraction and the black hole is apparently fully repulsive. In other words, the apparent probability that the rabbit will fall in the black hole during this time slot is zero. Moreover, since $\prod_{k=0}^{t} \tilde{p}(k)$ remains away from zero when $t \rightarrow \infty$, there will be a non zero asymptotic apparent probability that the rabbit never falls in the black hole during the black hole lifetime.

When $p=\rho$, we get $r_{1}(t)=t q p^{t-1}+p^{t}$ and $\tilde{p}(t)=1+O\left(\frac{1}{t}\right)$. Thus the rabbit as an apparent probability not to fall in the black hole during this time slot when $t \rightarrow \infty$. But since $\lim _{t \rightarrow \infty} \prod_{k=0}^{t} \tilde{p}(k)=$ 0 , the rabbit has an asymptotic apparent probability 1 to fall in the black hole during the black hole lifetime.

\subsection{Non unitary random walks}

In this section we investigate non unitary Markov systems with an infinite number of states. There is a specific state, the absorbing black hole state $s_{0}$ and there is an infinite sequence of states $s_{1}, s_{2}, \ldots, s_{n}, \ldots$. If the rabbit is in state $s_{0}$ its stay there for the remaining black hole lifetime, if the rabbit is in any of the states $s_{n}$, for $n>0$, then at the time unit it has probability $q$ to shift down to state $s_{n-1}$, and probability $p$ to shift up to state $s_{n+1}$ (see Figure 2). This is a nearest neighbor random walk in one dimension.

We denote $r_{n}(t)$ the probability sum when the rabbit is on state $s_{n}$ when the black hole has a remaining lifetime $t$. We denote $R_{n}(u)=\sum_{t \geq 0} r_{n}(t) u^{t}$ the generating function of the probability sum over all lifetimes $t$.

Let $t_{n}$ the time to black hole from state $s_{n}$, i.e. the time at which the rabbit hits the black hole when starting its journey at state $s_{n}$ at time 0 . The quantity $t_{n}$ is a random variable. Assuming the initial black hole lifetime $t$, when the rabbit enters the black hole it will experience a probability decay $\rho^{t-t_{n}}$, if $t_{n} \leq t$, 
otherwise it has no probability decay since we assume that the black hole loses its non unitarity properties after its evaporation. Therefore we have $r_{n}(t)=\sum_{\theta \leq t} P\left(t_{n}=\theta\right) \rho^{t-\theta}+P\left(t_{n}>t\right)$, and thus

$$
R_{n}(u)=F_{n}(u) \frac{1}{1-\rho u}+\left(1-F_{n}(u)\right) \frac{1}{1-u},
$$

with $F_{n}(u)$ the probability generating function of $t_{n} . F_{n}(u)$ satisfies $F_{n}(u)=q u F_{n-1}(u)+p u F_{n+1}(u)$, and therefore $F_{n}(u)=(F(u))^{n}$, with $1=\frac{q u}{F(u)}+p u F(u)$, which leads to

$$
F(u)=\frac{1-\sqrt{1-4 p q u^{2}}}{2 u p}
$$

This clearly mimics the fact that such walks can be seen as the product of $n$ consecutive Dyck paths, (reverse the time and cut on the last time the walk reach state $s_{n}$, for any $n>1$ ). Our aim is to evaluate the apparent repulsion $\tilde{p}_{n}(t)=p \frac{r_{n+1}(t-1)}{r_{n}(t)}$. Cauchy's integral formula gives

$$
r_{n}(t)=\frac{1}{2 \mathbf{i} \pi} \oint R_{n}(u) \frac{d u}{u^{t+1}},
$$

where $\mathbf{i}$ denotes the imaginary number $\mathbf{i}=\sqrt{-1}$. The probability sum generating function $R_{n}(u)$ has two main set of singularities, one single singularity at $u=\frac{1}{\rho}$, the inverse of the probability decay factor, and another pair of singularities at $\pm u(p)$ with $u(p)=\frac{1}{2 \sqrt{p q}}$. We expect a change in behavior when one set has smaller modulus than the other set. Notice that $u=1$ is not a singularity since $1-F_{n}(1)=0$ and by Lhospital rule, $R_{n}(u)$ can be analytically extended beyond $u=1$.

We now investigate the cases $\rho>2 \sqrt{p q}$ and $\rho<2 \sqrt{p q}$ which lead to very different asymptotic behavior of $\tilde{p}_{n}(t)$ when $t$ tends to infinity.

\subsubsection{Case $\rho>2 \sqrt{p q}$}

Theorem 2 We consider a one dimension, nearest neighbor, non unitary random walk with uniform switch up probability $p$ and switch down probability $q$, with a probability decay factor $\rho$ on the absorbing black hole state $s_{0}$. Let $t$ be the black hole remaining lifetime. Let $n$ be an integer $r$ a sequence of integer such that $n=o(\sqrt{t})$. Let $r_{n}(t)$ be the probability sum on state $s_{n}$, we define the apparent repulsion probability $\tilde{p}_{n}(t)$ as the ratio $\tilde{p}_{n}(t)=p \frac{r_{n+1}(t-1)}{r_{n}(t)}$, and the apparent attraction probabilities $\tilde{q}_{n}(t)=1-\tilde{p}_{n}(t)$. When the probability decay factor $\rho$ is greater than $2 \sqrt{p q}$, then we have $\tilde{q}_{n}(t)>\tilde{p}_{n}(t)$, i.e. the black hole is apparently attractive, for all states $s_{n}$ with $n>1$, when $t \rightarrow \infty$.

Proof: In this case $u=\frac{1}{\rho}$ is the dominant singularity in $R_{n}(u)$ and we choose an integral contour in equation $\sqrt{6}$, as follow. We take a circle centered on the origin with radius greater than $\frac{1}{\rho}$ and smaller than $u(p)$, so that the inscribed disk contains $\frac{1}{\rho}$ and exclude $\pm u(p)$ (see Figure 3 We take a radius $u(p)$, for all $u$ in this disk we have $|F(u)| \leq F(u(p))=\frac{1}{2 p u(p)}$. Using the residue theorem on the simple pole on $\frac{1}{\rho}$, we get the estimate

$$
r_{n}(t)=\left(F\left(\frac{1}{\rho}\right)\right)^{n} \rho^{t}+O\left(\frac{1}{(2 p)^{n}(u(p))^{n+t}}\right)
$$




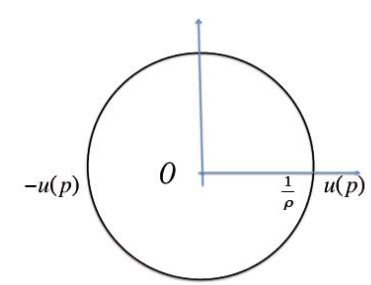

(a) $\frac{1}{\rho}<u(p)$, circle integral con-

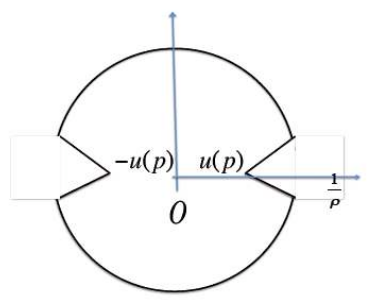

(b) $\frac{1}{\rho}>u(p)$, Hankel integral contour

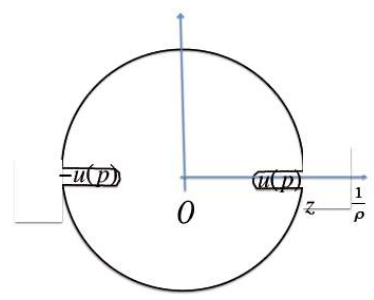

(c) $\frac{1}{\rho}>u(p)$, Integral contour for the unbounded values of $n$

Fig. 3:

and

$$
\tilde{p}_{n}(t)=\frac{p}{\rho} F\left(\frac{1}{\rho}\right)+O\left(\frac{(\rho u(p))^{-t}}{\left(2 p u(p) F\left(\frac{1}{\rho}\right)\right)^{n}}\right) .
$$

The error term exponentially tends to zero when $t \rightarrow \infty$, since $\rho u(p)>1$ and $n=o(\sqrt{t})$. We notice that $\lim \sup _{t \rightarrow \infty} \frac{\tilde{p}_{n}(t)}{p}>1$ since $1=\frac{q}{\rho F\left(\frac{1}{\rho}\right)}+\frac{p}{\rho} F\left(\frac{1}{\rho}\right)>\frac{p}{\rho} F\left(\frac{1}{\rho}\right)$.

\subsubsection{Case $\rho<2 \sqrt{p q}$}

Theorem 3 We consider a one dimension, nearest neighbor, non unitary walk with uniform move-up probability $p$ and move-down probability $q$, with a probability decay factor $\rho$ on the absorbing black hole state $s_{0}$. Let $t$ be the black hole remaining lifetime. Let $r_{n}(t)$ be the probability sum on state $s_{n}$ for an integer $n>0$, we define the apparent repulsion probability $\tilde{p}_{n}(t)$ as the ratio $\tilde{p}_{n}(t)=p \frac{r_{n+1}(t-1)}{r_{n}(t)}$, and the apparent attraction probabilities $\tilde{q}_{n}(t)=1-\tilde{p}_{n}(t)$. When the probability decay factor $\rho$ is smaller than $2 \sqrt{p q}$, then we have $\tilde{q}_{n}(t)<\tilde{p}_{n}(t)$, i.e. the black hole is apparently repulsive for all state $s_{n}$ with $n>1$, when $t \rightarrow \infty$ as soon as $n=o(\sqrt{t})$.

Proof: In this case the set $\left\{ \pm \frac{1}{2 \sqrt{p q}}\right\}$ contains the dominant singularities of the probability sum generating function $R_{n}(u)$. Let $u(p)=\frac{1}{2 \sqrt{p q}}$. When $u= \pm(u(p)+\delta u)$ we have

$$
F^{n}(u)=\frac{1}{(2 u p)^{n}}\left(1-2 n(p q)^{\frac{1}{4}} \sqrt{\delta u}+O\left(n^{2} \delta u\right)\right)
$$

We take advantage of the fact that $R_{n}(u)$ has an explicit form as a polynomial function of $\sqrt{1-\frac{u^{2}}{u^{2}(p)}}$, for which there are important established results about the asymptotic analysis of their coefficients due to Flajolet and Odlyzko. Thus we will use a didactic approach where we first start with the simpler case of a bounded value of $n$, (i.e. which does not change with $t$ ), and second we terminate the proof with the more intricate case where $n$ is unbounded (and increases with $t$ such that $n=o(\sqrt{t})$ ). 
Bounded values of $n \quad$ Applying the Flajolet Odlyzko [3] asymptotic result, namely

$$
\begin{aligned}
& r_{n}(t)=\frac{\rho^{n+t}}{(2 p)^{n}}+\sqrt{\pi} \frac{n}{t^{\frac{3}{2}}} \frac{(p q)^{\frac{1}{4}}}{(2 p)^{n}(u(p))^{n+t}} \\
& \left.\times\left(\frac{1}{1-\rho u(p)}-\frac{1}{1-u(p)}+\frac{(-1)^{n+t}}{1+\rho u(p)}\right)-\frac{(-1)^{n+t}}{1+u(p)}+O\left(\frac{n}{t}\right)\right) .
\end{aligned}
$$

The result is based on an Hankel integral contour made of a circle of radius $u(p)+\varepsilon<\frac{1}{\rho}$ indented by two wedges on $\pm u(p)$ (see Figure 3). Notice the oscillations that appear between the odd and even values of $n+t$ which are due to the model artefact that the random is nearest neighbor only. These oscillations cancel on the values of the apparent repulsion $\tilde{p}_{n}(t)$ for $n>1$ which satisfies:

$$
\tilde{p}_{n}(t)=\frac{p r_{n+1}(t-1)}{p r_{n+1}(t-1)+q r_{n-1}(t-1)}=\frac{1}{2} \frac{n+1}{n}\left(1+O\left(\frac{1}{t}\right)+O\left(\frac{n}{t}\right)\right)
$$

since $r_{n+1}(t-1)=\frac{q}{p} r_{n-1}(t-1)\left(1+O\left(\frac{1}{t}\right)+O\left(\frac{n}{t}\right)\right)$. Thus the apparent repulsion $\tilde{p}_{n}(t)$ converges to $\frac{1}{2}$ as soon as $t \rightarrow \infty$ with $\frac{n}{t} \rightarrow 0$. In other words the apparent random random walk tends to be neutral with attraction balancing the repulsion.

For $n=1$, since $r_{0}(t)=\rho^{t} \ll r_{1}(t)=O\left(\frac{1}{\left.u_{(}\right)^{t}}\right)$ we have $\tilde{p}_{1}(t) \rightarrow 1$ : the black hole is apparently $100 \%$ repulsive on the last state before, and the random walk on the remaining states is apparently neutral. In other words the random walk is apparently neutral and reflective on the last state before the black hole.

Unbounded values of $n$ Here we consider unbounded values of $n$, but with the restriction that $n=$ $o(\sqrt{t})$ when $t \rightarrow \infty$. We nevertheless need a more involved analysis on the Cauchy identity:

$$
r_{n}(t)=\frac{1}{2 \mathbf{i} \pi(2 p)^{n}} \oint\left(1-\sqrt{1-4 p q u^{2}}\right)^{n} g_{\rho}(u) \frac{d u}{u^{n+t+1}}
$$

with $g_{\rho}(u)=\left(\frac{1}{1-\rho u}-\frac{1}{1-u}\right)$. We deform the Hankel integral loop to a circle of radius $z>u(p)$, indented by two pairs of segments parallel to the real axis that connect the circle to the internal singularities $\pm u(p)$ and encircle them (see Figure 3. We get for any arbitrary $z<\frac{1}{\rho}$

$$
r_{n}(t)=I_{n}(t, z)+J_{n}(t, z)+O\left(\frac{1}{(2 p)^{n} z^{n+t}}\right)
$$

with

$$
\begin{aligned}
I_{n}(t, z) & =\frac{1}{2 \mathbf{i} \pi(2 p)^{n}} \int_{u(p)}^{z}\left(\left(1+\mathbf{i} \sqrt{4 p q u^{2}-1}\right)^{n}-\left(1-\mathbf{i} \sqrt{4 p q u^{2}-1}\right)^{n}\right) g_{\rho}(u) \frac{d u}{u^{n+t+1}} \\
J_{n}(t, z) & =\frac{-1}{2 \mathbf{i} \pi(2 p)^{n}} \int_{-z}^{-u(p)}\left(\left(1+\mathbf{i} \sqrt{4 p q u^{2}-1}\right)^{n}-\left(1-\mathbf{i} \sqrt{4 p q u^{2}-1}\right)^{n}\right) g_{\rho}(u) \frac{d u}{u^{n+t+1}}(15)
\end{aligned}
$$


With change of variable $u=\left(1+\frac{v}{n+t}\right) u(p)$, we get

$$
\begin{aligned}
u^{n+t+1} & =\left(1+O\left(\frac{v^{2}}{n+t+1}\right)\right) \exp (v) \\
\left(1+\mathbf{i} \sqrt{4 p q u^{2}-1}\right)^{n} & =\left(1+O\left(\frac{v n}{n+t}\right)\right) \exp \left(\mathbf{i} \frac{n}{\sqrt{n+t+1}} \sqrt{2 v}\right) \\
\left(1-\mathbf{i} \sqrt{4 p q u^{2}-1}\right)^{n} & =\left(1+O\left(\frac{v n}{n+t}\right)\right) \exp \left(-\mathbf{i} \frac{n}{\sqrt{n+t+1}} \sqrt{2 v}\right) \\
g_{\rho}(u) & =\left(1+O\left(\frac{v}{n+t}\right)\right) g_{\rho}(u(p))
\end{aligned}
$$

and consequently

$$
\begin{aligned}
I_{n}(t, z)= & \frac{g_{\rho}(u(p))}{(n+t) \pi(2 p)^{n} u(p)^{n+t+1}}\left(\int_{0}^{(z-u(p)) \frac{n+t+1}{u(p)}} \sin \left(\frac{n}{\sqrt{n+t}} \sqrt{2 v}\right) e^{-v} d v+O\left(\frac{n}{n+t}\right)\right) \\
& \frac{g_{\rho}(u(p))}{(n+t) \pi(2 p)^{n} u(p)^{n+t+1}}\left(\sqrt{\frac{\pi}{2}} \frac{n}{\sqrt{n+t}} \exp \left(-\frac{1}{2} \frac{n^{2}}{n+t}\right)+O\left(\frac{n}{n+t}\right)\right)
\end{aligned}
$$

Similarly with the change of variable $u=-\left(1+\frac{v}{n+t}\right) u(p)$ we get

$$
J_{n}(t, z)=\frac{g_{\rho}(-u(p))(-1)^{n+t+1}}{(n+t) \pi(2 p)^{n} u(p)^{n+t+1}}\left(\sqrt{\frac{\pi}{2}} \frac{n}{\sqrt{n+t}} \exp \left(-\frac{1}{2} \frac{n^{2}}{n+t}\right)+O\left(\frac{n}{n+t}\right)\right)
$$

which validates the previous asymptotic estimates obtained via the Flajolet-Odlyzko method on bounded values of $n$, and extend them to the case where $n$ is unbounded but with the restriction $n=o(\sqrt{t})$.

\subsection{Random walk potential}

We call the quantity $V_{n}=n \frac{1}{2} \log \frac{q}{p}$ the potential of the random walk at state $s_{n}$. Clearly the state $s_{0}$ is the state with the lowest potential. We notice that $V_{n+1}-V_{n}$ is equal to the opposite of the logarithm of $\sqrt{\frac{p}{q}}$. If $p$ and $q$ were depending on $n$ we should have the random walk locally attractive when $V_{n+1}-V_{n}>0$, or locally repulsive when $V_{n+1}-V_{n}<0$. Notice that these considerations do not change if we add to the potential an arbitrary constant.

Noticing that the ratio of apparent attraction probability over the apparent repulsion probability satisfies $\frac{\tilde{q}_{n}(t)}{\tilde{p}_{n}(t)}=\frac{q}{p} \frac{r_{n-1}(t-1)}{r_{n+1}(t-1)}$, the apparent potential $\tilde{V}_{n}(t)=\sum_{i \leq n} \frac{1}{2} \log \frac{\tilde{q}_{i}(t)}{\tilde{p}_{i}(t)}$ is

$$
V_{n}-\frac{1}{2} \log r_{n+1}(t-1)-\frac{1}{2} \log r_{n}(t-1) \text {. }
$$

It comes from the above section analysis that when $\frac{1}{\rho}<u(p)$ (equivalent to the case $\rho>2 \sqrt{p q}$ ) we have an apparent potential $\tilde{V}_{n}(t)$ which tends to be equal to $n \frac{1}{2} \log \frac{1-\tilde{p}}{\tilde{p}}$, modulo an arbitrary constant term, with $\tilde{p}=\frac{p}{\rho} F\left(\frac{1}{\rho}\right)$, and in this case state $s_{0}$ is the state with the lowest potential. The black hole is 
still attractive. When $\frac{1}{\rho}<u(p)$ (equivalent to the case $\rho>2 \sqrt{p q}$ ), the apparent potential tends to be flat, except for a peak on the black hole state. Figure 4 and 5 show the asymptotic apparent potentials when $\rho$ is above or below $2 \sqrt{p q}$. Notice that as expected the apparent repulsion does not change very much as soon as $\rho<2 \sqrt{p q}$.

\section{Non unitary concave walk}

We call a concave walk a one dimension, nearest neighbor, random walk where the transition pair of probabilities $p_{n}, q_{n}$ depends on the state and $p_{n}$ increases with $n$ with $\lim p_{n} \leq \frac{1}{2}$ when $n \rightarrow \infty$ (see Figure 6. For example $p_{n}=\frac{1}{2}-\frac{\beta}{n^{2}}$ if one wants to simulate the random walk of a particle around a gravitationally heavy object such as a stellar body. Or $p_{n}=\frac{1}{2}-\frac{\beta}{n}$ if one wants to simulate the random walk of a particle in a gravitationally heavy medium of density decreasing in the inverse of distance to the center (state $s_{0}$ ). A gravitational walk can be used to simulate the behavior of a particle in a gas where each collision with another particle give a random momentum.

Our aim is to guess the behavior of the random walk with respect to its tuning parameter. In particular we will show when the random walk is stable and conjecture in the general case, that the random walk is apparently repulsive when the probability decay factor $\rho$ is smaller than $\lim _{n \rightarrow \infty} 2 \sqrt{p_{n} q_{n}}$, and is attractive when the probability is greater than this threshold. When the probability decay factor is within $o\left(\frac{1}{\sqrt{t}}\right)$ to this threshold, quantity $t$ being the black hole lifetime, then the random walk is apparently bimodal: i.e. it is apparently attractive within a certain state $B$ and is repulsive beyond that state.

Similarly as with uniform walk we define the actual potential of the random walk as

$$
V_{n}=\sum_{j=1}^{j=n} \frac{1}{2} \log \left(\frac{q_{j}}{p_{j}}\right) .
$$

The step down time of state $s_{i}$ is the time needed for the rabbit starting on state $s_{i}$ to arrive on state $s_{i-1}$ just below. The step down time is a random variable and let $G_{i}(u)$ be the probability generating function of the step down time of state $s_{i}$. Since the time $t_{n}$ is equal to the sum of the step down times of respective states $s_{n}, s_{n-1}$, etc., and since these step down times are independent, we have

$$
F_{n}(u)=\prod_{i=1}^{i=n} G_{i}(u)
$$

Let $n$ be an integer greater than 0 . Since from state $s_{n}$ the rabbit during a single time step can only access its nearest neighbor states, $s_{n-1}$ and $s_{n+1}$ with respective probabilities $q_{n}$ and $p_{n}$, we have the recursion

$$
F_{n}(u)=q_{n} u F_{n-1}(u)+p_{n} u F_{n+1}(u),
$$

or

$$
G_{n}(u)=q_{n} u+p_{n} u G_{n+1}(u) G_{n}(u) .
$$

We therefore get the recursion

$$
G_{n}(u)=\frac{q_{n} u}{1-p_{n} u G_{n+1}(u)}
$$




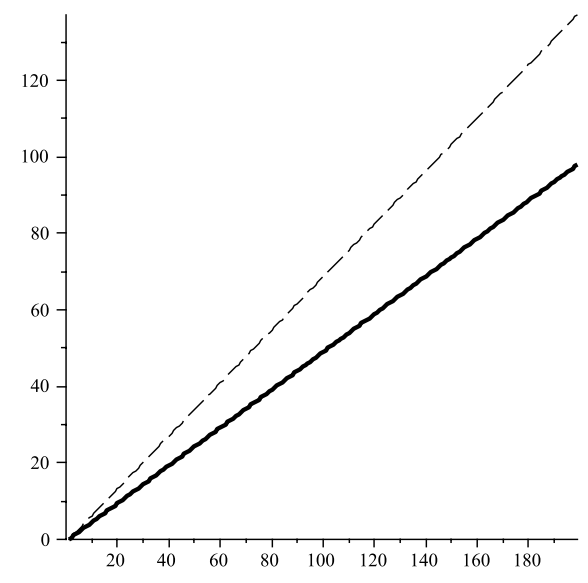

(a) $\rho=0.9$

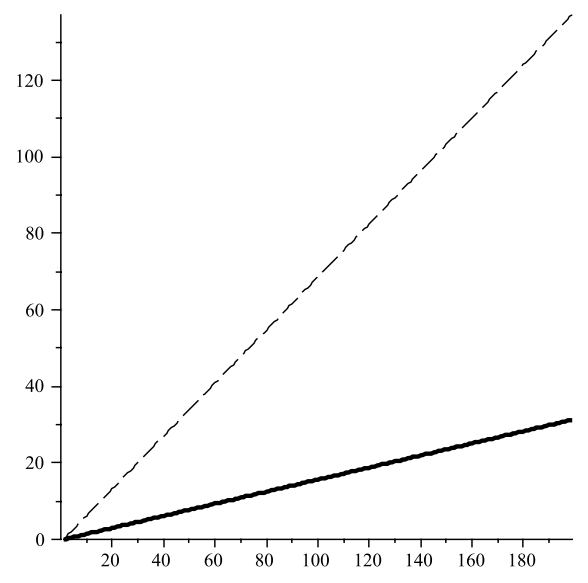

(b) $\rho=0.81$

Fig. 4: Actual potential (dashed) and apparent asymptotic potential (plain) for $p=0.2$, two values for $\rho$ greater than $2 \sqrt{p q}=0.8$

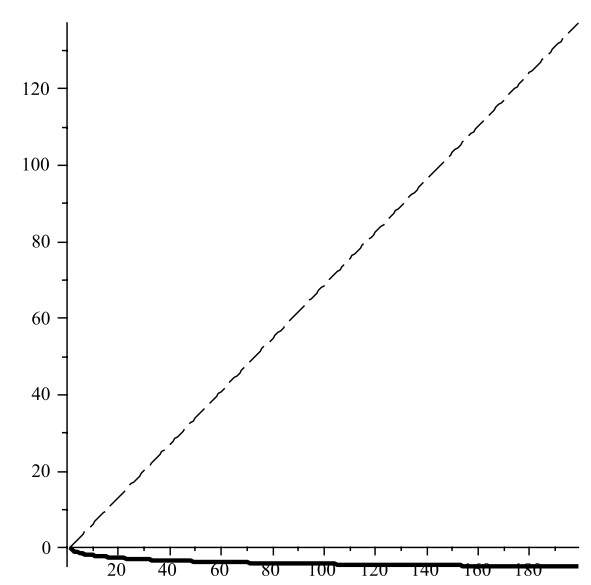

(a) $\rho=0.79$

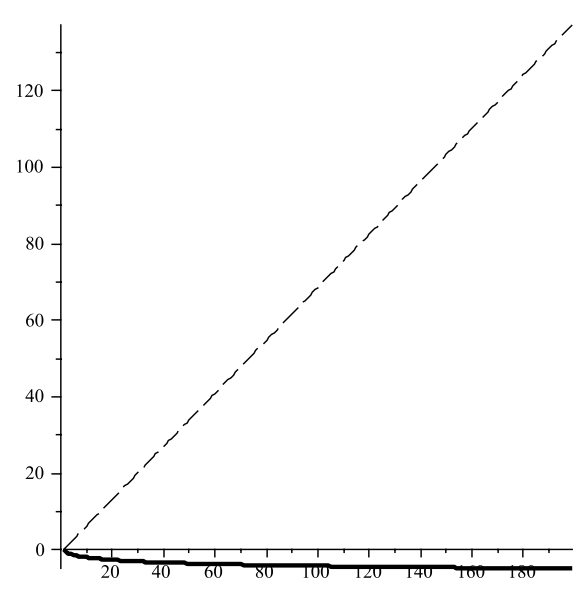

(b) $\rho=0.3$

Fig. 5: Actual potential (dashed) and apparent asymptotic potential (plain) for $p=0.2$, two values for $\rho$ smaller than $2 \sqrt{p q}=0.8$. 


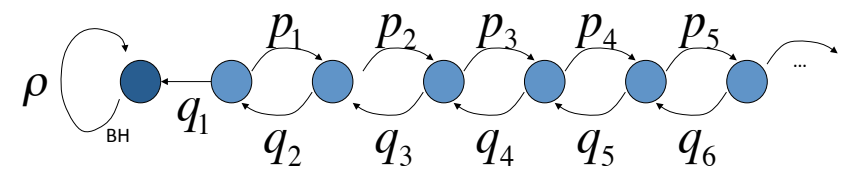

Fig. 6: Non unitary concave random walk.

which expands into the classic continuous fraction as noticed in [1]:

$$
G_{n}(u)=\frac{q_{n} u}{1-\frac{p_{n} q_{n+1} u^{2}}{1-\frac{p_{n+1} q_{n+2} u^{2}}{1-\cdots}}} .
$$

We notice that for all $n$, function $G_{n}(u)$ is odd: $G_{n}(-u)=-G_{n}(u)$. Let us denote $F(u, p)=$ $\frac{1-\sqrt{1-4 p q u^{2}}}{2 p u}$. We have for all $u>1$ real:

$$
F\left(u, p_{n}\right) \leq G_{n}(u) \leq F\left(u, p_{\infty}\right) .
$$

\subsection{Quasi-continuous walk}

We consider that we are in quasi-continuity conditions when the values $p_{n}$ does not vary quickly. For example $p_{n}=p(\alpha n)$ where $p($.$) is a continuous function and \alpha$ is a small non negative real number. In quasi-continuity condition function $G_{n}($.$) is close to the fixed point of the functional equation G_{n}(u)=$ $\frac{q_{n} u}{1-p_{n} u G_{n}(u)}$, or in other words and uniformly in $n$ and $u$ in a compact neighborhood of zero:

$$
\lim _{\alpha \rightarrow 0} G_{n}(u)=F\left(u, p_{n}\right)=\frac{1-\sqrt{1-4 p_{n} q_{n} u^{2}}}{2 p_{n} u} .
$$

In fact we can prove that the convergence holds for all complex numbers $u$ such that $\lim _{n \rightarrow \infty} \sup 4 p_{n} q_{n}|u|^{2} \leq$ 1 , for which values the function $G_{n}(u)$ are all analytical.

\subsection{Stable walks}

We will assume that there exists an integer $N$ such that the random walk probabilities take a fixed value $p_{\infty}$ beyond state $s_{N}: \forall n \geq N, p_{n}=p_{\infty}$. Therefore for all $n \geq N: G_{n}=\frac{1-\sqrt{1-4 p_{\infty} q_{\infty} u^{2}}}{2 p_{\infty} u}$.

Let $u_{n}=2 \sqrt{p_{n} q_{n}}$ and $\bar{u}=u_{N}$. The main singularity of $G_{N}(u)$ is $\bar{u}$ since $\forall n \geq N: G_{n}(u)=$ $G_{N}(u)=\frac{1}{2 p_{\infty} u}\left(1-D_{N} \sqrt{1-\frac{u^{2}}{\bar{u}^{2}}}\right)$ for some $D_{N}$, for instance $D_{N}=1$. Using recursion 26, we see that $\{ \pm \bar{u}\}$ is also the main singularity set of $G_{n}(u)$ for $n<N$ as we see below.

\subsubsection{Properties of $G_{n}(u)$}

Let $s(u)=1-\frac{u^{2}}{\bar{u}^{2}}$. Let $K_{n}(u)$ denotes $2 p_{n} u G_{n}(u)$, the reduced step down generating function of state $s_{n}$. Notice that $K_{n}(u)$ is an even function: $K_{n}(-u)=K_{n}(u)$ for all complex numbers $u$. We have the 
recursion

$$
K_{n}(u)=\frac{2 p_{n} q_{n} u^{2}}{1-\frac{p_{n}}{2} p_{n+1} K_{n+1}(u)}
$$

We notice that $K_{n}(u)$ is an algebraic function, this can be proven by descending recursion from $n=N$ and we can split

$$
K_{n}(u)=H_{n}(u)-\sqrt{s(u)} Q_{n}(u)
$$

with $H_{n}(u)$ and $Q_{n}(u)$ analytical fractions defined and bounded for $|u|<|\bar{u}|+\varepsilon$ for some $\varepsilon>0$. Notice that there is only one possible decomposition of $K_{n}(u)$, and we call $H_{n}(u)$ the rational part of function $K_{n}(u)$, and $Q_{n}(u)$ the algebraic part of function $K_{n}(u)$. When $n \geq N$, we have $K_{n}(u)=1-\sqrt{s(u)}$ : $H_{n}(u)=Q_{n}(u)=1$. From recursion 30, we get

$$
\begin{aligned}
H_{n}(u) & =2 p_{n} q_{n} u^{2} \frac{1}{1-\frac{p_{n}}{2 p_{n+1}} H_{n+1}(u)-\frac{p_{n}}{2 p_{n+1}} \sqrt{s(u)} Q_{n+1}(u)} \\
& =2 p_{n} q_{n} u^{2} \frac{1-\frac{p_{n}}{2 p_{n+1}} H_{n+1}(u)+\frac{p_{n}}{2 p_{n+1}} \sqrt{s(u)} Q_{n+1}(u)}{\left(1-\frac{p_{n}}{2 p_{n+1}} H_{n+1}(u)\right)^{2}+\left(\frac{u^{2}}{\bar{u}^{2}}-1\right)\left(\frac{p_{n}}{2 p_{n+1}} Q_{n+1}(u)\right)^{2}}
\end{aligned}
$$

Theorem 4 We consider a stable concave walk such that for all $n \geq N,\left(p_{n}, q_{n}\right)=\left(p_{\infty}, q_{\infty}\right)$. Let $\bar{u}=\frac{1}{2 \sqrt{p_{\infty} q_{\infty}}}$. For $n>0$ let $G_{n}(u)$ be the probability generating function of the step down time from state $s_{n}$ to state $s_{n-1}$. Let $K_{n}(u)=2 p_{n} u G_{n}(u)$ the reduced step down generating function. Let $s(u)=1-\frac{u^{2}}{\bar{u}^{2}}$, and the unique decomposition

$$
K_{n}(u)=H_{n}(u)-\sqrt{s(u)} Q_{n}(u)
$$

the rational functions $H_{n}(u)$ and $Q_{n}(u)$ being respectively the rational and algebraic parts of $K_{n}(u)$.

There exists $\varepsilon>0$ such that for all integers $n$, both the rational and algebraic parts of the generating function $K_{n}(u)$ are uniformly bounded for all complex number $u$ such that $|u|<\bar{u}+\varepsilon$.

Basically we need to prove the theorem for $n \leq N$. We formally identify $K_{N}(u)$ with the variable $w$ and define the bivariate step down generating function $K_{n}(u, w)$ via the recursion

$$
K_{n}(u, w)=\frac{2 p_{n} q_{n} u^{2}}{1-\frac{p_{n}}{2 q_{n+1}} K_{n+1}(u, w)}
$$

The function $K_{n}(u, w)$ is analytical and has positive Taylor coefficients. We keep in mind the identity

$$
K_{n}(u)=K_{n}(u, 1-\sqrt{s(u)})
$$

Lemma 1 There exists $\varepsilon>0$ such that for all complex numbers $u$ such that $|u| \leq \bar{u}+\varepsilon$, for all complex numbers $w$ such that $|w| \leq 1+\varepsilon$ and for all integer $n$, the bivariate reduced step down generating function satisfies $\left|K_{n}(u, w)\right| \leq 1+\varepsilon$. 
Proof: We notice that $2 p_{n} q_{n}=\frac{u_{n}^{2}}{2}$ is an increasing function of $n$. We also have $\frac{p_{n}}{p_{n+1}} \leq 1$. Let $n<N$, there exist $\varepsilon>0$ such that if $|u| \leq \bar{u}+\varepsilon$ and $\left|K_{n+1}(u, w)\right| \leq 1+\varepsilon$, then $\left|K_{n}(u, w)\right| \leq 1+\varepsilon$. Indeed from recursion (32):

$$
\left|K_{n}(u, w)\right| \leq\left|4 p_{n} q_{n} u^{2}\right| \frac{1}{2-\left|K_{n+1}(u, w)\right|} \leq\left|4 p_{n} q_{n} u^{2}\right| \frac{1}{1-\varepsilon},
$$

and this we set $|u|<u_{N-1}$ thus, $\left|4 p_{n} q_{n} u^{2}\right| \leq \frac{|u|^{2}}{u_{N-1}^{2}} \leq 1-\varepsilon^{2}$, for some $\varepsilon$ such that $|u| \leq \bar{u}+\varepsilon$.

Proof of Theorem 4: Now, we have to express the rational and algebraic parts, $H_{n}(u)$ and $Q_{n}(u)$, with the help of the bivariate function $K_{n}(u, w)$. Let consider $K_{n}(u, 1-x)$, we denote $H_{n}(u, y)$ the $x$-even part of $K_{n}(u, 1-x)$, namely $H_{n}\left(u, x^{2}\right)=\frac{1}{2}\left(K_{n}(u, 1-x)+K_{n}(u, 1+x)\right)$ and $Q_{n}(u, y)$ the $x$-odd part of $K_{n}(u, 1-x)$, namely $\frac{-1}{2 x}\left(K_{n}(u, 1-x)-K_{n}(u, 1+x)\right)$. Both are analytical in $u$ and $y$ and we have the relation

$$
\left\{\begin{array}{l}
H_{n}(u)=H_{n}\left(u, 1-\frac{u^{2}}{\bar{u}^{2}}\right) \\
Q_{n}(u)=Q_{n}\left(u, 1-\frac{u^{2}}{\bar{u}^{2}}\right) .
\end{array}\right.
$$

It remains to prove that $H_{n}\left(u, x^{2}\right)$ and $Q_{n}\left(u, x^{2}\right)$ are bounded. This is provided by the integral representation

$$
\left\{\begin{array}{l}
H_{n}(u, y)=\frac{1}{2 \mathbf{i} \pi} \oint z H_{n}(u, 1-z) \frac{d z}{z^{2}-y} \\
Q_{n}(u, y)=\frac{1}{2 \dot{i} \pi} \oint H_{n}(u, 1-z) \frac{d z}{z^{2}-y},
\end{array}\right.
$$

with appropriate integral loops around the complex number $y$ such that $|1-z|<1+\varepsilon$. These last identities give the formal proof that the $H_{n}(u)$ and $Q_{n}(u)$ are analytical and uniformly bounded for $|u| \leq \bar{u}+\varepsilon$ for some $\varepsilon>0$. This terminates the proof of Theorem 4 .

Theorem 5 We consider a stable concave walk such that for all $n \geq N,\left(p_{n}, q_{n}\right)=\left(p_{\infty}, q_{\infty}\right)$. Let $\bar{u}=\frac{1}{2 \sqrt{p_{\infty} q_{\infty}}}$. For $n>0$ let $G_{n}(u)$ be the probability generating function of the step down time from state $s_{n}$ to state $s_{n-1}$. Let $s(u)=1-\frac{u^{2}}{\bar{u}^{2}}$. There exists a complex neighborhood of $\{ \pm \bar{u}\}$ such that for all complex numbers $u$ in this complex neighborhood, the three following points hold for all integers $n \leq N$ :

(i) the logarithm of the step down time generating function $\left.G_{n}(u)\right)$ exists and is well defined,

(ii) in the unique algebraic decomposition

$$
\log G_{n}(u)=h_{n}(u)-\sqrt{s(u)} q_{n}(u),
$$

where $h_{n}(u)$ and $q_{n}(u)$ are analytical functions defined in the complex neighborhood of $\{ \pm \bar{u}\}$, both functions are uniformly bounded,

(iii) the function $q_{n}(u)$ uniformly decays exponentially when $n$ decreases.

The proof will need the following lemmas with their corollaries.

Lemma 2 For all complex numbers $u$ such that $|u| \leq \bar{u}+\varepsilon$ and for all complex numbers $w$ such that $|w| \leq 1+\varepsilon$, and for all integers $n<N$ the bivariate step down generating function satisfies $\left|K_{n}(u, w)\right| \leq$ $\min \left\{4 p_{n} \bar{u}^{2}, 1\right\}(1+\varepsilon)$ 
Proof: We just reuse recursion 32 and notice that, when $n<N$,

$$
\begin{aligned}
\left|K_{n}(u, w)\right| & \leq \frac{p_{n} q_{n}}{p_{N-1} q_{N-1}} 4 p_{N-1} q_{N-1}|u| \frac{1}{2-\left|K_{n+1}(u, w)\right|} \\
& \leq \frac{p_{n} q_{n}}{p_{N-1} q_{N-1}}(1+\varepsilon) .
\end{aligned}
$$

We end the proof of the lemma with the fact that $\frac{p_{n} q_{n}}{p_{N-1} q_{N-1}} \leq \frac{p_{n} q_{n}}{p_{\infty} q_{\infty}}=4 p_{n} q_{n} \bar{u}^{2} \leq 4 p_{n} \bar{u}^{2}$.

Corollary 1 We consider a stable concave walk such that for all $n \geq N,\left(p_{n}, q_{n}\right)=\left(p_{\infty}, q_{\infty}\right)$. Let $\bar{u}=\frac{1}{2 \sqrt{p_{\infty} q_{\infty}}}$. For $n>0$ let $G_{n}(u)$ be the probability generating function of the step down time from state $s_{n}$ to state $s_{n-1}$. Let $K_{n}(u)=2 p_{n} u G_{n}(u)$ the reduced step down generating function. Let $s(u)=1-\frac{u^{2}}{\bar{u}^{2}}$, and the unique decomposition

$$
K_{n}(u)=H_{n}(u)-\sqrt{s(u)} Q_{n}(u)
$$

the rational functions $H_{n}(u)$ and $Q_{n}(u)$ being respectively the rational and algebraic parts of $K_{n}(u)$. For all complex numbers $u$ such that $|u| \leq \bar{u}+\varepsilon$ and for all $n \leq N$ the functions $\frac{1}{p_{n}} H_{n}(u)$ and $\frac{1}{p_{n}} Q_{n}(u)$, are uniformly bounded.

Lemma 3 For all complex numbers $u$ such that $|u| \leq \bar{u}$ and for all integers $n<N$, the reduced step down generating functions satisfy $\left|K_{n}(u)\right| \leq \frac{p_{n}}{p_{\infty}} \leq 1$.

Proof: When $|u| \leq \bar{u}$ we have $\left|G_{n}(u)\right| \leq G_{n}(\bar{u}) \leq \frac{1}{2 p_{\infty} \bar{u}}$.

Lemma 4 For all $\varepsilon>0$, there exists a neighborhood of $\bar{u}$ such that for all complex numbers $u$ in this neighborhood, and for all integers $n<N$, the algebraic part of the reduced step down generating function, $Q_{n}(u)$, exponentially decays when $n$ decreases and satisfies $\left|Q_{n}(u)\right| \leq Q_{n}^{1-\varepsilon}(\bar{u})$.

Proof: We rewrite recursion (30) with

$$
\begin{aligned}
& H_{n}(u)=\frac{1}{2 p_{n} q_{n} u^{2}} K_{n}(u) \bar{K}_{n}(u)\left(1-\frac{p_{n}}{2 p_{n+1}} H_{n+1}(u)\right) \\
& Q_{n}(u)=\frac{1}{4 p_{n+1} q_{n} u^{2}} K_{n}(u) \bar{K}_{n}(u) Q_{n+1}(u)
\end{aligned}
$$

with $\bar{K}_{n}(u)=H_{n}(u)+\sqrt{s(u)} Q_{n}(u)$.

Furthermore, since $\frac{1}{p_{n}} K_{n}(u)=\frac{1}{p_{n}} H_{n}(u)-\sqrt{s(u)} \frac{1}{p_{n}} Q_{n}(u), \frac{1}{p_{n}} H_{n}(u)$ and $\frac{1}{p_{n}} Q_{n}(u)$, are uniformly bounded for all $n \leq N$, thanks to Cauchy theorem, they are also uniformly continuous. Since $s(\bar{u})=0$, for any $\varepsilon>0$ there exists a neighborhood of $\bar{u}$ such that $\left|K_{n}(u)\right|<\frac{p_{n}}{p_{\infty}}(1+\varepsilon)$, uniformly for all $n \leq N$. Similarly, since $\bar{K}_{n}(u)=K_{n}(u)+2 \sqrt{s(u)} Q_{n}(u)$ we also have a neighborhood of $\bar{u}$ where 
$\left|\bar{K}_{n}(u)\right| \leq \frac{p_{n}}{p_{\infty}}(1+\varepsilon)$. Consequently for $u$ in this neighborhood (assuming $|u-\bar{u}|<\varepsilon \bar{u}$ ), we have

$$
\begin{aligned}
\left|Q_{n}(u)\right| & =\frac{1}{4 p_{n+1} p_{n}|u|^{2}}\left|K_{n}(u) \bar{K}_{n}(u)\right|\left|Q_{n+1}(u)\right| \\
& \leq \frac{1}{4 p_{n+1} p_{n}|u|^{2}} \frac{p_{n}^{2}}{p^{2}}(1+\varepsilon)^{2}\left|Q_{n+1}(u)\right| \\
& \leq \frac{1}{4 p_{n+1} p_{n} \bar{u}^{2}} \frac{p_{n}^{2}}{p_{\infty}^{2}}(1+\varepsilon)^{2} \frac{\bar{u}^{2}}{|u|^{2}}\left|Q_{n+1}(u)\right| \\
& \leq \frac{4 p_{\infty} q_{\infty}}{4 p_{n+1} q_{n}} \frac{p_{n}^{2}}{p_{\infty}^{2}}(1+\varepsilon)^{4}\left|Q_{n+1}(u)\right| \\
& =\frac{p_{n}}{p_{n+1}} \frac{q_{\infty}}{q_{n}} \frac{p_{n}}{p_{\infty}}(1+\varepsilon)^{4}\left|Q_{n+1}(u)\right| \leq \frac{q_{\infty}}{q_{n}} \frac{p_{n}}{p_{\infty}}(1+\varepsilon)^{4}\left|Q_{n+1}(u)\right| .
\end{aligned}
$$

Therefore $Q_{n}(u)$ decays exponentially like $\prod_{j=n}^{j=N-1} \frac{q_{\infty}}{q_{j}} \frac{p_{j}}{p_{\infty}}(1+\varepsilon)^{4}$ as soon as $\frac{q_{\infty}}{q_{n}} \frac{p_{n}}{p_{\infty}}(1+\varepsilon)^{4}<1$.

Lemma 5 For all integers $n$, we have the inequality $H_{n}(\bar{u})=K_{n}(\bar{u})>2 p_{n} \bar{u}$.

Proof: we have $K_{n}(\bar{u})=2 p_{n} \bar{u} G_{n}(\bar{u})$ and also the fact that $G_{n}(\bar{u}) \geq 1$.

Proof of Theorem 5: We prove the theorem for the neighborhood of $\bar{u}$. The proof for the neighborhood of $-\bar{u}$ comes by symmetry. Since $\frac{1}{p_{n}} K_{n}(\bar{u})$ is uniformly bounded from below and $\frac{1}{p_{n}} H_{n}(u)$ is uniformly bounded from above and is continuous around $\bar{u}$, and since $K_{n}(\bar{u})=H_{n}(\bar{u})$, there exists a neighborhood of $\bar{u}$ where $\frac{1}{p_{n}} K_{n}(u)$ and $\frac{1}{p_{n}} H_{n}(u)$ are non zero and uniformly bounded from above and from below. If this neighborhood is a simple disk, then $\log \frac{1}{p_{n}} K_{n}(u)$ and $\log \frac{1}{p_{n}} H_{n}(u)$ exist. We also have the identity

$$
\log \left(\frac{1}{p_{n}} K_{n}(u)\right)=h_{n}(u)-\sqrt{s(u)} q_{n}(u) .
$$

Since

we have

$$
\begin{aligned}
& \frac{1}{p_{n}} H_{n}(u)-\sqrt{s(u)} \frac{1}{p_{n}} Q_{n}(u)=e^{h_{n}(u)} e^{-\sqrt{s(u)} q_{n}(u)} \\
& \frac{1}{p_{n}} H_{n}(u)+\sqrt{s(u)} \frac{1}{p_{n}} Q_{n}(u)=e^{h_{n}(u)} e^{\sqrt{s(u)} q_{n}(u)}
\end{aligned}
$$

$$
\begin{aligned}
h_{n}(u) & =\log \left(\frac{1}{p_{n}} H_{n}(u)\right)+\frac{1}{2} \log \left(1-s(u) \frac{Q_{n}^{2}(u)}{H_{n}^{2}(u)}\right) \\
q_{n}(u) & =\frac{1}{2 \sqrt{s(u)}} \log \frac{H_{n}(u)-\sqrt{s(u)} Q_{n}(u)}{H_{n}(u)+\sqrt{s(u)} Q_{n}(u)} .
\end{aligned}
$$

Notice that $q_{n}(u)$ is of order $\frac{Q_{n}(u)}{H_{n}(u)}$ and decays exponentially as $Q_{n}(u)$ when $n$ decreases. This terminates the proof of Theorem 5

The following theorem will be used in order to get an acurate estimate of the remaining terms in the evaluation of the probability sums in the non unitary concave walks.

Theorem 6 We consider a stable concave walk such that for all $n \geq N,\left(p_{n}, q_{n}\right)=\left(p_{\infty}, q_{\infty}\right)$. Let $\bar{u}=\frac{1}{2 \sqrt{p_{\infty} q_{\infty}}}$. For $n>0$ let $G_{n}(u)$ be the step down time generating function from state $s_{n}$ to state $s_{n-1}$. Let $K_{n}(u)=2 p_{n} u G_{n}(u)$ the reduced step down generating function. There exists $\varepsilon>0$ and $a$ real number $\beta>0$ such that for all $n \leq N$ and for all complex numbers $u$ such $|u-\bar{u}| \leq \varepsilon$, the logarithm of the algebraic part $Q_{n}(u)$ of the generating function $K_{n}(u)$ exists and the following inequality holds: $\left|\log \frac{Q_{n}(u)}{Q_{n}(\bar{u})}\right| \leq(N-n)|u-\bar{u}| \beta$ 
Proof: We rewrite again the recursion

$$
\frac{Q_{n}(u)}{Q_{n+1}(u)}=\frac{1}{4 p_{n+1} q_{n} u^{2}} K_{n}(u) \bar{K}_{n}(u) .
$$

Since for all $n<N, \log K_{n}(u)$ and $\log \bar{K}_{n}(u)$ exists, thus $\log \frac{Q_{n}(u)}{Q_{n+1}(u)}$ exists. Since $\log K_{n}(u)$ and $\log \bar{K}_{n}(u)$ are unformly bounded on a complex neighborhood of $\bar{u}$, we have the existence of $\beta>0$ such that for all complex numbers $u$ in this neighborhood:

$$
\left|\log \frac{K_{n}(u) \bar{K}_{n}(u)}{u^{2}}-\log \frac{\left(K_{n}(\bar{u})\right)^{2}}{\bar{u}^{2}}\right| \leq \beta|u-\bar{u}| .
$$

Remember in passing that $K_{n}(\bar{u})=\bar{K}_{n}(\bar{u})$. Therefore it comes that

$$
\left|\log \frac{Q_{n}(u)}{Q_{n+1}(u)}-\log \frac{Q_{n}(\bar{u})}{Q_{n+1}(\bar{u})}\right| \leq \beta|u-\bar{u}| .
$$

The logarithm of $Q_{n}(u)$ exists since it is equal to the $\operatorname{sum} \sum_{i=n}^{N} \log \frac{Q_{i}(u)}{Q_{i+1}(u)}\left(\right.$ since $\left.Q_{N}(u)=1\right)$ and the following inequality holds:

$$
\left|\log Q_{n}(u)-\log Q_{n}(\bar{u})\right| \leq(N-n)|u-\bar{u}| \beta .
$$

We denote $D_{n}=q_{n}(\bar{u})$, the value of the algebraic part of the logarithm of the step down generating function at $\bar{u}$. We have

$$
D_{n}=\frac{p_{n}}{q_{n}} G_{n}(\bar{u}) G_{n+1}(\bar{u}) D_{n+1} .
$$

Quasi-continuous concave walk In this subsection we export the above results in the case of quasicontinuous random walk as defined in Subsection 4.1. We have the asymptotic estimate.

$$
\log F_{n}(u)=\sum_{j=1}^{n} \log G_{j}(u)=\frac{1}{\alpha} \int_{0}^{\alpha n} \log F(u, p(x)) d x+O(1),
$$

and

$$
\log D_{n}=\sum_{j=1}^{n} \log \frac{p_{j}}{q_{j}} G_{j}(\bar{u}) G_{j+1}(\bar{u})=\frac{1}{\alpha} \int_{\alpha n}^{\infty} \log \left(\frac{p(x)}{q(x)} F^{2}(\bar{u}, p(x))\right) d x+O(1) .
$$

\subsection{Behavior of non unitary concave stable walks}

\subsubsection{Case where $\rho>2 \sqrt{p_{\infty} q_{\infty}}$}

This is the simplest of the three cases. The inverse of the probability decay factor $\frac{1}{\rho}$ is the main singularity and leads to a simple pole. Quantities $\pm \bar{u}$ are the secondary singularities whose contribution will be detailed in the next section. If we assume that $\frac{1}{\rho}<z<\bar{u}$ we get

$$
r_{n}(t)=F_{n}\left(\frac{1}{\rho}\right) \rho^{-t}+O\left(F_{n}(z) \frac{z^{-t}}{1-\rho z}\right) .
$$




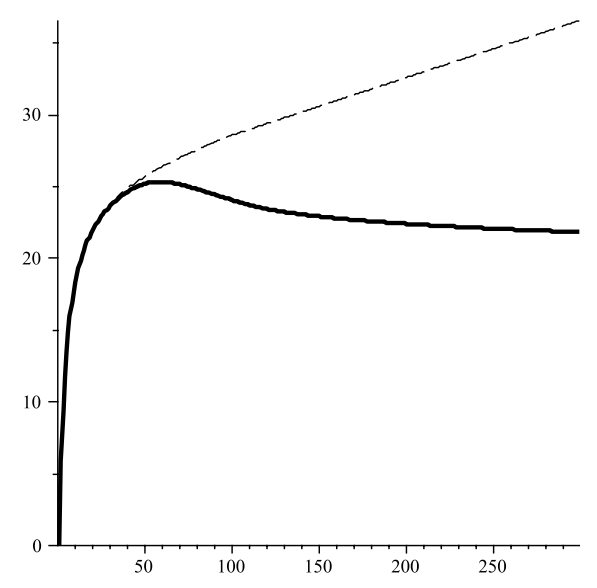

Fig. 7: Actual potential (dashed) and apparent asymptotic potential (plain) for a concave walk with $p_{n}=$ $0.48 \exp \left(-\frac{100}{n^{2}}\right), N=100, t=10,000, \rho=\exp (0.0005) \bar{u}^{-1}$.

With $F_{n}(u)=\prod_{j=1}^{j=n} G_{j}(u)$ we get

$$
\tilde{p}_{n}(t)=p_{n} \frac{1}{\rho} G_{n+1}\left(\frac{1}{\rho}\right)+O\left((\rho z)^{-t}\right) .
$$

In quasi-continuity condition we have or all $u<u_{n}: G_{n}(u)=F(u, p(\alpha n))+O(\alpha)$ and therefore we get the result we had for uniform random walk that is

$$
\tilde{p}_{n}(t)=p(\alpha n) \frac{1}{\rho} F\left(\frac{1}{\rho}, p(\alpha n)\right)+O(\alpha)+O\left((\rho z)^{-t}\right) .
$$

Figure 7 shows the apparent potential when $\rho \bar{u}>1$.

\subsubsection{Case where $\rho<2 \sqrt{p_{\infty} q_{\infty}}$}

Theorem 7 We consider a stable non unitary concave walk such that for all $n \geq N\left(p_{n}, q_{n}\right)=\left(p_{\infty}, q_{\infty}\right)$, with a probability decay factor $\rho$ on the absorbing black hole state $s_{0}$. Let $t$ be the black hole remaining lifetime. Let $\bar{u}=\frac{1}{2 \sqrt{p_{\infty} q_{\infty}}}$. When the probability decay factor satisfies the condition $\rho \bar{u}<1$, we have the asymptotic estimate of the probability sum at state $s_{n}$ for black hole lifetime $t$, when $t \rightarrow \infty$ as soon as $n, N=o(\sqrt{t})$ :

$$
\begin{aligned}
r_{n}(t)= & \sqrt{\pi} \frac{F_{n}(\bar{u})}{(n+t)^{\frac{3}{2}}} \frac{\left(p_{\infty} q_{\infty}\right)^{\frac{1}{4}}}{(\bar{u})^{t}}\left(\sum_{j=1}^{j=n} D_{j}\right) \\
& \left(g_{\rho}(\bar{u})+(-1)^{n+t} g_{\rho}(-\bar{u})\right)\left(1+O\left(\frac{N}{n+t}\right)\right)+O\left((1+\varepsilon)^{n} z^{-t}\right),
\end{aligned}
$$

with $F_{n}(u)$ being the generating function of the time to black hole from state $s_{n}$, $t_{n}$, and is equal to the product of the step down generating functions up to integer $n: F_{n}(u)=\prod_{j=1}^{j=n} G_{j}(u)$. 
Proof: We define $z=(1+\varepsilon) \bar{u}$ which is the upper-limit of the radius where $|u| \leq z$ implies $\left|K_{n}(u)\right|<$ $1+\varepsilon$. In this section we assume that $\frac{1}{\rho}>z$, i.e. the disk $|u| \leq z$ does not contain any other singularities than $\pm \bar{u}$ for $F_{n}(u) g_{\rho}(u)$.

We have

$$
F_{n}(u)=\prod_{j=1}^{j=n} G_{j}(u)=\frac{1}{u^{n}} \exp \left(\sum_{j=1}^{j=n} h_{j}(u)-\sqrt{s(u)} q_{j}(u)\right)
$$

We have $h_{n}(\bar{u})=\log \left(\bar{u} G_{n}(\bar{u})\right)$. Let $D_{n}=q_{n}(\bar{u})=\frac{Q_{n}(\bar{u})}{H_{n}(\bar{u})}$. We have the expression

$$
D_{n}=\frac{p_{n}}{q_{n}} G_{n}(\bar{u}) G_{n+1}(\bar{u}) D_{n+1}
$$

which gives $D_{n}=\prod_{j=n}^{j=N} \frac{p_{j}}{q_{j}} G_{j}(\bar{u}) G_{j+1}(\bar{u})$. Notice that $\frac{p_{n}}{q_{n}} G_{n}(\bar{u}) G_{n+1}(\bar{u}) \leq 1$ since $\frac{p_{n}}{q_{n}} \leq \frac{p_{\infty}}{q_{\infty}}$

$$
\frac{p_{n}}{q_{n}} G_{n}(\bar{u}) G_{n+1}(\bar{u}) \leq \frac{p_{\infty}}{q_{\infty}} F^{2}\left(\bar{u}, p_{\infty}\right)=\frac{p_{\infty}}{q_{\infty}} \frac{1}{4\left(p_{\infty}\right)^{2} \bar{u}^{2}}=1
$$

Our aim is to find an accurate estimate of $r_{n}(t)$ via the Cauchy formula

$$
r_{n}(t)=\frac{1}{2 \mathbf{i} \pi} \oint \prod \frac{K_{j}(u)}{p_{j}} \frac{d u}{u^{n+t}} g_{\rho}(u)
$$

As in the previous section we have the estimate

$$
r_{n}(t)=I_{n}(t, z)+J_{n}(t, z)+O\left((1+\varepsilon)^{n} z^{-t}\right) .
$$

The key of our analysis is the estimate of $I_{n}(z, t)$ and $J_{n}(z, t)$. This is an integration with the factor $u^{n} F_{n}(u)=\exp \left(\sum_{j=1}^{j=n} h_{j}(u)-\sqrt{s(u)} q_{j}(u)\right)$. By doing the change of variable $u=\left(1+\frac{v}{n+t}\right) \bar{u}$ we get

$$
\begin{aligned}
\frac{1}{u^{t}} F_{n}(u)= & \frac{1}{\bar{u}^{n+t}} \exp \left(\sum_{j=1}^{j=n} h_{j}(\bar{u})+\frac{1}{n+t} O_{1}(v)-\sqrt{-2 v} \frac{D_{j}}{\sqrt{n+t}}\right. \\
& \left.+\sqrt{-2 v} \frac{1}{(n+t)^{\frac{1}{2}}} O_{2}\left(\left(e^{(N-j)|u-\bar{u}| \beta}-1\right) D_{j}\right)\right) e^{-v} \\
= & \frac{1}{\bar{u}^{t}} \prod_{j=1}^{j=n} G_{j}(\bar{u})\left(1-\sqrt{-2 v} \frac{\sum_{j=1}^{j=n} D_{j}}{\sqrt{n+t}}+\frac{n}{n+t} O_{1}^{\prime}(v)\right. \\
& \left.+\sqrt{-2 v} \frac{1}{(n+t)^{\frac{3}{2}}} \sum_{j=1}^{j=n} O_{2}^{\prime}\left(v D_{j}(N-j)\right)\right) e^{-v}
\end{aligned}
$$


Quantity $\beta$ is given by Theorem 6 Notice that $O_{1}(v)$ and $O_{1}^{\prime}(v)$ are both analytic in $v$ with the domain of definition including the integration paths. Therefore about the expression of $I_{n}(t, z)$ we have

$$
\begin{aligned}
I_{n}(t, z)= & \frac{1}{2 \mathbf{i} \pi} \int_{\bar{u}}^{z}\left(\exp \left(\sum_{j=1}^{j=n} h_{j}(u)+\mathbf{i} q_{j}(u) \sqrt{\frac{u^{2}}{\bar{u}}-1}\right)\right. \\
& \left.-\exp \left(\sum_{j=1}^{j=n} h_{j}(u)-\mathbf{i} q_{j}(u) \sqrt{\frac{u^{2}}{\bar{u}}-1}\right)\right) g_{\rho}(u) \frac{d u}{u^{n+t+1}} \\
= & \frac{\bar{u}^{-t}}{\pi} \prod_{j=1}^{j=n} G_{j}(\bar{u})\left(\sum_{j=1}^{j=n} D_{j} \sqrt{2 v} e^{-v}+\sum_{j=1}^{j=n} O_{2}^{\prime}\left(D_{j}\right) \frac{N}{(n+t)^{\frac{3}{2}}}\right)+O\left((1+\varepsilon)^{n} z^{-t}\right) .
\end{aligned}
$$

Notice that the term in $O_{1}^{\prime}(v) \frac{n}{n+t}$ disappears when we substract the term in $-\mathbf{i} \sqrt{\frac{u^{2}}{\bar{u}^{2}}-1}$ :

$$
\begin{aligned}
r_{n}(t)= & \sqrt{\pi} \frac{F_{n}(\bar{u})}{(n+t)^{\frac{3}{2}}} \frac{\left(p_{\infty} q_{\infty}\right)^{\frac{1}{4}}}{(\bar{u})^{t}}\left(\sum_{j=1}^{j=n} D_{j}\right) \\
& \left(g_{\rho}(\bar{u})+(-1)^{n+t} g_{\rho}(-\bar{u})\right)\left(1+O\left(\frac{N}{n+t}\right)\right)+O\left((1+\varepsilon)^{n} z^{-t}\right) .
\end{aligned}
$$

With $F_{n}(u)=\prod_{j=1}^{j=n} G_{j}(u)$, we get the following evaluation of the apparent repulsion:

$$
\tilde{p}_{n}(t)=p_{n} G_{n+1}(\bar{u}) u_{N} \frac{\sum_{j=1}^{j=n+1} D_{j}}{\sum_{j=1}^{j=n} D_{j}}\left(1+O\left(\frac{N}{n+t}\right)\right) .
$$

In quasi-continuity conditions we can identify $G_{n}(u)$ with $F\left(u, p_{n}\right)$ and $D_{n+1-k}=\left(\frac{p_{n}}{q_{n}} F^{2}\left(u, p_{n}\right)\right)^{k} D_{n+1}$. This leads to $\sum_{j=1}^{j=n+1} D_{j}=\frac{1}{1-\frac{p_{n}}{q_{n}} F^{2}\left(u, p_{n}\right)} D_{n+1}$ and $\sum_{j=1}^{j=n} D_{j}=\frac{\frac{p_{n}}{q_{n}} F^{2}\left(u, p_{n}\right)}{1-\frac{p_{n}}{q_{n}} F^{2}\left(u, p_{n}\right)} D_{n+1}$

$$
\lim _{\alpha \rightarrow 0, t \rightarrow \infty} \tilde{p}_{n}(t)=\frac{\bar{u}}{F\left(\bar{u}, p_{n}\right)} q_{n} .
$$

It turns out that the black hole is simply repulsive since $\tilde{p}_{n}(t) \geq\left(u_{N}\right)^{2} 2 p_{n} q_{n}=\frac{1}{2}\left(1+\sqrt{1-\left(\frac{\bar{u}}{u_{n}}\right)^{2}}\right) \geq$ $\frac{1}{2}$, and the closer we are to the black hole, the stronger is the repulsion. When $n>N$ we get naturally $\tilde{p}_{n}(t) \approx \frac{1}{2}$ : the random walk is apparently neutral beyond the state $s_{N}$ where the coefficients are stable.

In the limit case where $u_{N} \approx 1$, we would have $\tilde{p}_{n}(t) \approx q_{n}$ : the random walk is apparently strictly reversed.

Corollary 2 We consider a stable non unitary concave walk such that for all $n \geq N,\left(p_{n}, q_{n}\right)=$ $\left(p_{\infty}, q_{\infty}\right)$, with a probability decay factor $\rho$ on the absorbing black hole state $s_{0}$. Let $t$ be the black hole remaining lifetime. Let $\bar{u}=\frac{1}{2 \sqrt{p_{\infty} q_{\infty}}}$. We have the asymptotic relation between the probability sum 
$r_{n}(t)$ at state $s_{n}$, the probability decay factor $\rho$ and the probability distribution of the time $t_{n}$ to black hole from state $s_{n}: r_{n}(t)=P\left(t_{n}=t\right)\left(\frac{1}{1-\rho \bar{u}}+\frac{(-1)^{n+t}}{1+\rho \bar{u}}\right)\left(1+O\left(\frac{N}{n+t}\right)\right)+P\left(t_{n}>t\right)$, when $t \rightarrow \infty$ as soon as $n, N=o(\sqrt{t})$.

\subsubsection{Case where $\rho \approx 2 \sqrt{p_{\infty} q_{\infty}}$}

Case $\frac{1}{\rho}<\bar{u}$

Theorem 8 We consider a stable non unitary concave walk such that for all $n \geq N,\left(p_{n}, q_{n}\right)=\left(p_{\infty}, q_{\infty}\right)$ with a probability decay factor $\rho$ on the absorbing black hole state $s_{0}$. Let t be the black hole remaining lifetime. Let $\bar{u}=\frac{1}{2 \sqrt{p_{\infty} q_{\infty}}}$. Let $z$ be an arbitrary real number such $z>\bar{u}$. There exists $\varepsilon>0$ such that when $t \rightarrow \infty$ ans as soon as $n, N=o(\sqrt{t})$, we have the asymptotic relation between the probability sum $r_{n}(t)$ at state $s_{n}$, the probability decay factor $\rho$ and the probability generating function $F_{n}(u)$ of the time to black hole from state $s_{n}$ :

$$
\begin{aligned}
r_{n}(t)= & \rho^{t} F_{n}\left(\frac{1}{\rho}\right)+\bar{u}^{-t} \sqrt{\pi} F_{n}(\bar{u}) \frac{\sum_{j=1}^{j=n} D_{j}}{(n+t)^{\frac{3}{2}}}\left(p_{\infty} q_{\infty}\right)^{\frac{1}{4}}\left(g_{\rho}(\bar{u})+(-1)^{n+t} g_{\rho}(-\bar{u})\right)\left(1+O\left(\frac{N}{n+t}\right)\right) \\
& +O\left((1+\varepsilon)^{n} z^{-t}\right),
\end{aligned}
$$

where $D_{n}$ is the value of the algebraic part of the step down generating function at $\bar{u}$.

Proof: We assume that $\frac{1}{\rho}-\bar{u}=\frac{1}{o(t)}=o\left(\frac{1}{\sqrt{t}}\right)$. We use an integral contour like in Figure 8 It suffices to add the contribution of both $\frac{1}{\rho}$ and $\bar{u}$ :

$$
\begin{aligned}
r_{n}(t)= & \rho^{t} F_{n}\left(\frac{1}{\rho}\right)+\bar{u}^{-t} \sqrt{\pi} F_{n}(\bar{u}) \frac{\sum_{j=1}^{j=n} D_{j}}{(n+t)^{\frac{3}{2}}}\left(p_{\infty} q_{\infty}\right)^{\frac{1}{4}}\left(g_{\rho}(\bar{u})+(-1)^{n+t} g_{\rho}(-\bar{u})\right)\left(1+O\left(\frac{N}{n+t}\right)\right) \\
& +O\left((1+\varepsilon)^{n} z^{-t}\right) .
\end{aligned}
$$

Notice that the term in $\bar{u}^{-t}$ is always smaller than the term in $\rho^{t}$.

Case $\frac{1}{\rho}>\bar{u}$ and $\left.\rho \bar{u} \in\right] 1-o\left(\frac{1}{\sqrt{t}}\right), 1-\frac{1}{o(t)}[$

Theorem 9 We consider a stable non unitary concave walk such that for all $n \geq N,\left(p_{n}, q_{n}\right)=\left(p_{\infty}, q_{\infty}\right)$ with a probability decay factor $\rho$ on the absorbing black hole state $s_{0}$. Let $\bar{u}=\frac{1}{2 \sqrt{p_{\infty} q_{\infty}}}$. When the probability decay factor $\rho$ is such that the product $\rho \bar{u}$ belongs to an interval of the kind $] 1-o\left(\frac{1}{\sqrt{t}}\right), 1-\frac{1}{o(t)}[$, we have the estimate, when $t \rightarrow \infty$ as soon as $n, N=o(\sqrt{t})$ :

$$
\begin{aligned}
\frac{r_{n}(t)}{F_{n}(\bar{u})}= & \rho^{t}+\bar{u}^{-t} \sqrt{\pi} \frac{\sum_{j=1}^{j=n} D_{j}}{(n+t)^{\frac{3}{2}}}\left(p_{\infty} q_{\infty}\right)^{\frac{1}{4}}\left(g_{\rho}(\bar{u})+(-1)^{n+t} g_{\rho}(-\bar{u})\right) \\
& +O\left(\frac{1}{\sqrt{\frac{1}{\rho}-\bar{u}}}\right) \rho^{t} \sum_{j=1}^{j=n} D_{j} .
\end{aligned}
$$

where $F_{n}(u)$ is the probability generating function of the time to black hole from state $s_{n}$, and $D_{n}$ is the value of the algebraic part of the step down generating function at $\bar{u}$.

Proof: This case is interesting because the second main singularity $\frac{1}{\rho}$ stands right in the integration path of $I_{n}(t, z)$, and $g_{\rho}(u)$ becomes singular at $u=\frac{1}{\rho}$. To remove this annoying conjunction we bend 


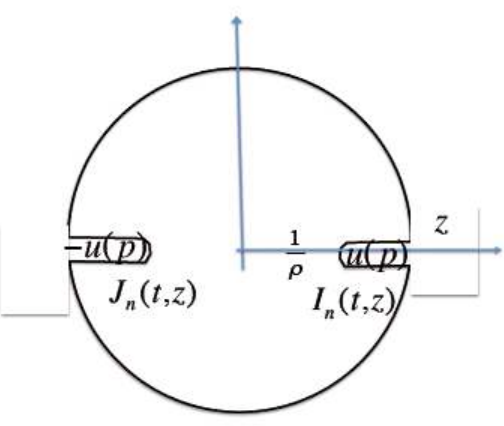

(a) $\frac{1}{\rho}>u(p)$, integral contour

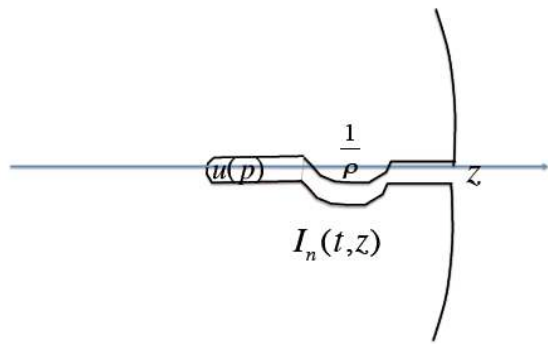

(b) $\frac{1}{\rho}<u(p)$, detail of the integral contour

Fig. 8: Case $\rho \bar{u} \approx 1$.

the integration path of $I_{n}(t, z)$ so that it avoids the point $\frac{1}{\rho}$ and therefore the singularity at $u=\frac{1}{\rho}$ becomes a simple pole (see Figure 8). Anyhow the detour will introduce a correction term of order $F_{n}(\bar{u}) g_{\rho}(\bar{u}) \sum_{j=1}^{n} D_{j} \sqrt{1-\frac{1}{(\rho \bar{u})^{2}}} \rho^{t}$. That is an error term in $F_{n}(\bar{u}) \rho^{t} O\left(\frac{1}{\sqrt{\frac{1}{\rho}-\bar{u}}}\right) \sum_{j=1}^{n} D_{j}$.

We assume that $\frac{1}{\rho}-\bar{u}$ is both $o\left(\frac{1}{\sqrt{t}}\right)$ and $\frac{1}{o(t)}$. We have $F_{n}\left(\frac{1}{\rho}\right)=F_{n}(\bar{u})\left(1+O\left(\frac{n}{\sqrt{t}}\right) \sum_{j=1}^{j=n} D_{j}\right)$. Therefore we get

$$
\begin{aligned}
\frac{r_{n}(t)}{F_{n}(\bar{u})}= & \rho^{t}+\bar{u}^{-t} \sqrt{\pi} \frac{\sum_{j=1}^{j=n} D_{j}}{(n+t)^{\frac{3}{2}}}\left(p_{\infty} q_{\infty}\right)^{\frac{1}{4}}\left(g_{\rho}(\bar{u})+(-1)^{n+t} g_{\rho}(-\bar{u})\right) \\
& +O\left(\frac{1}{\sqrt{\frac{1}{\rho}-\bar{u}}}\right) \rho^{t} \sum_{j=1}^{j=n} D_{j} .
\end{aligned}
$$

Notice that $g_{\rho}(\bar{u})=O\left(\frac{1}{\frac{1}{\rho}-\bar{u}}\right)=o(t)$ and the error term is negligible in front of the term in $\bar{u}^{-t}$. Since $F_{n}\left(\frac{1}{\rho}\right)=F_{n}(\bar{u})\left(1+O\left(\frac{n}{\sqrt{t}}\right)\right)$ and $\sum_{j=1}^{j=n+1} D_{j}=\frac{1}{1-\frac{p_{n}}{q_{n}} F^{2}\left(\bar{u}, p_{n}\right)} D_{n+1}$ in quasi continuous conditions, we get (removing the error term)

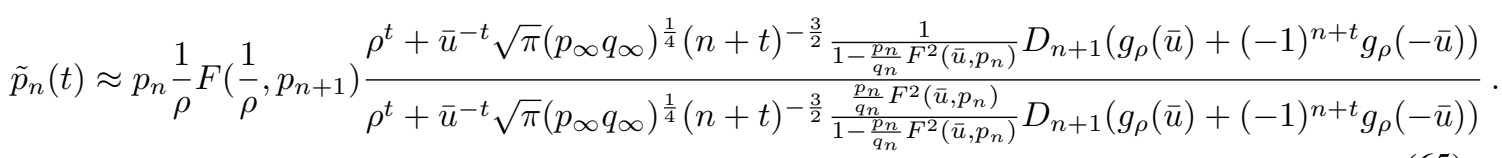

We cannot say that any of the two terms in $\rho^{t}$ and in $\bar{u}^{-t}$ is negligible in front of the other one.

Corollary 3 In quasi continuous conditions we have $p_{n}=p(\alpha n)$ where $p(x)$ is a fixed continuous function. Let $q(x)=1-p(x)$. We assume that $\alpha \rightarrow 0$. When $t \rightarrow \infty$ there is a state $s_{B}$ such that the black hole is attractive before this state and attractive beyond, and we have the estimate $B=\frac{z}{\alpha}$ with $z$ a real number such that

$$
\int_{z}^{\infty} \log \left(\frac{p(x)}{q(x)} F^{2}(\bar{u}, p(x))\right) d x=\alpha \log \left(\rho^{t} \bar{u}^{t} t^{\frac{3}{2}}(1-\rho \bar{u})\right) .
$$


Proof: We observe that if we define integer $B$ such that

$$
\rho^{t} \approx \bar{u}^{-t} \sqrt{\pi}\left(p_{\infty} q_{\infty}\right)^{\frac{1}{4}} \frac{1}{1-\frac{p_{B}}{q_{B}} F^{2}\left(\bar{u}, p_{B}\right)} D_{B+1}\left(g_{\rho}(\bar{u})+(-1)^{n+t} g_{\rho}(-\bar{u})\right) .
$$

When $n<B$ then the term in $\rho^{t}$ will be preponderant and in this case

$$
\tilde{p}_{n}(t) \approx p_{n} \frac{1}{\rho} F\left(\frac{1}{\rho}, p_{n}\right)
$$

which means that the black hole is attractive. When $n>B$, then

$$
\tilde{p}_{n}(t) \approx q_{n} \frac{1}{\rho F\left(\frac{1}{\rho}, p_{n}\right)},
$$

in other words, the black hole is repulsive beyond state $s_{B}$. Notice that the change of mode is sharp: there is a briskly change of the value of $\tilde{p}_{n}(t)$ in a small set of contiguous states, leading to an edge in the apparent random walk potential (see Figure 9 and following).

Notice that $\tilde{p}_{n}(t) \rightarrow \frac{1}{2}$ when $n$ increases: the random walk is asymptotically neutral.

Ignoring $O(\alpha)$ terms, the change of mode occurs on state $s_{B}$ with $B=\left\lfloor\frac{z}{\alpha}\right\rfloor$ such that

$$
\int_{z}^{\infty} \log \left(\frac{p(x)}{q(x)} F^{2}(\bar{u}, p(x))\right) d x=\alpha \log \left(\rho^{t} \bar{u}^{t} t^{\frac{3}{2}}(1-\rho \bar{u})\right) .
$$

Figures 9 and 10 shows the apparent potential of a concave stable walk with different $\rho<\bar{u}^{-1}$.

\subsection{Generalized concave walks}

In this subsection we don't consider anymore that the random walk coefficient are stable beyond a fixed step $N$. In this case we simply assume that $\lim p_{n}=p_{\infty}$ and we denote $\bar{u}=u\left(p_{\infty}\right)$.

The main difficulty is in the convergence of the decomposition functions $K_{n}(u)=H_{n}(u)-\sqrt{s(u)} Q_{n}(u)$. In passing we notice that the decomposition functions are no longer rational, but only analytical defined in a complex neighborhood of both $\pm \bar{u}$. Therefore we call $H_{n}(u)$ and $Q_{n}(u)$ respectively the analytical and algebraic part of generating function $K_{n}(u)$. Therefore we assume that the series $\sqrt{p_{\infty}-p_{n}}$ converge. We can prove that there exists $\varepsilon>0$ and $N$ such for all $u$ with $|u|<\bar{u}(1+\varepsilon)$ and for all $n>N$ :

$$
K_{n}(u, v)=v+O\left(\sum_{j \geq n} p_{\infty}-p_{j}\right)
$$

A more thorough analysis shows that Lemma 1 can be extended to the statement that when $|u|<u_{N}$ the following bounding condition holds: $\left|K_{N}(u, v)\right| \leq 1+\sqrt{1-\frac{|u|^{2}}{u_{N}^{2}}}$. To this end we assume that $\left.1-\frac{|u|^{2}}{u_{N}^{2}} \leq(1-\varepsilon) \sqrt{1-\frac{\bar{u}^{2}}{u_{N}^{2}}} \leq(1-\varepsilon) \sqrt{2\left(p_{\infty}-p_{N}\right)}\right)$. But $\sum_{j \geq n} p_{\infty}-p_{j}=o\left(\sqrt{p_{\infty}-p_{n}}\right.$. Therefore by letting $N$ large enough such that $\left|K_{N}(u, v)\right| \leq 1+\sqrt{1-\frac{|u|^{2}}{u_{N}^{2}}}$, we can export the results of stable random concave walks to general concave walk. 


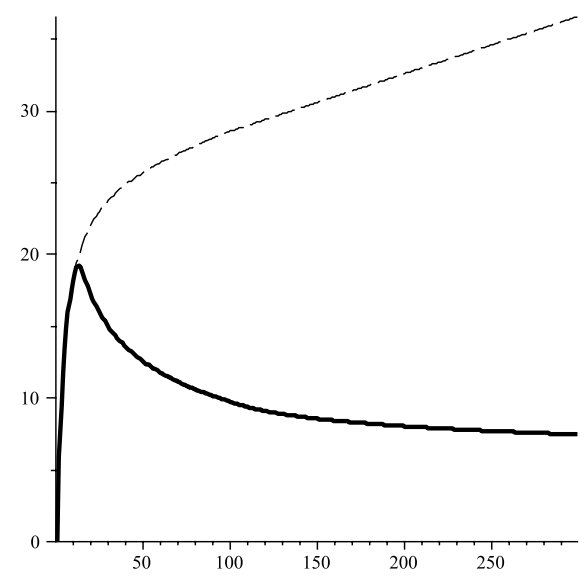

(a) $\rho=\exp (-0.002) \bar{u}^{-1}$

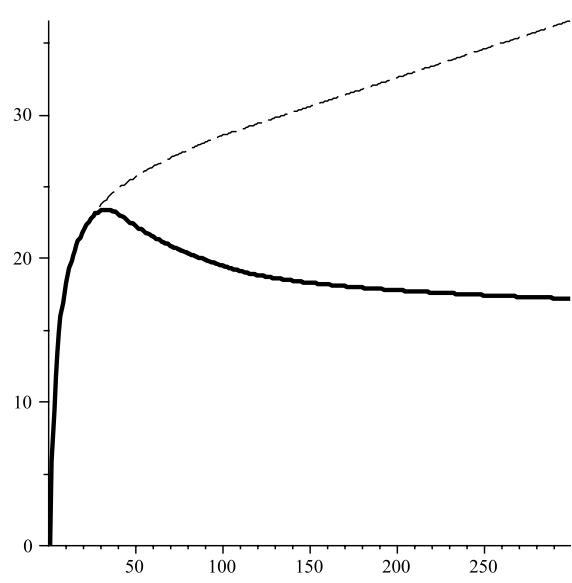

(b) $\rho=\exp (-0.001) \bar{u}^{-1}$

Fig. 9: Actual potential (dashed) and apparent asymptotic potential (plain) for a concave walk with $p_{n}=$ $0.48 \exp \left(-\frac{100}{n^{2}}\right), N=100$ and $t=10,000$.

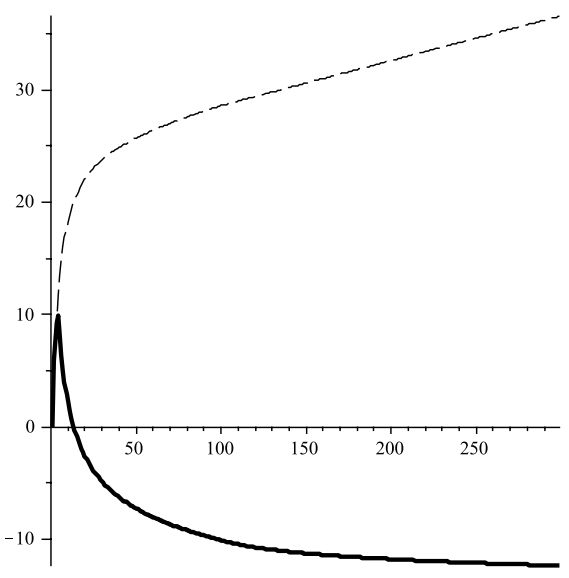

(a) $\rho=\exp (-0.004) \bar{u}^{-1}$

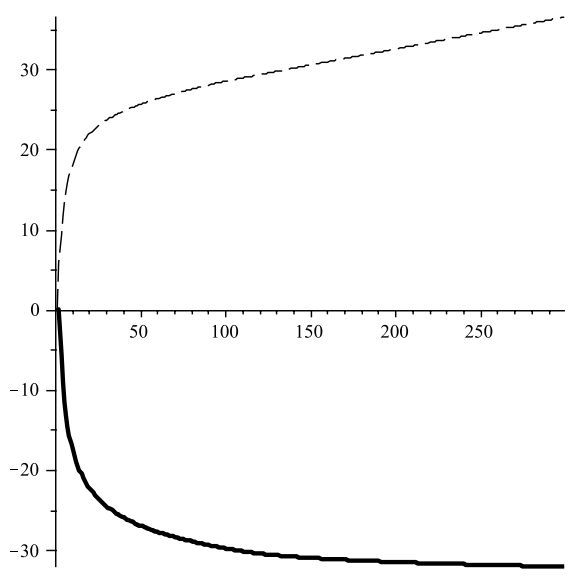

(b) $\rho=\exp (-0.006) \bar{u}^{-1}$

Fig. 10: Actual potential (dashed) and apparent asymptotic potential (plain) for a concave walk with $p_{n}=$ $0.48 \exp \left(-\frac{100}{n^{2}}\right), N=100$ and $t=10,000$. 


\section{Non unitary Gravitational walks}

We call gravitational walk a concave walk where $p_{\infty}=\frac{1}{2}$. Or, in other words $\bar{u}=1$. For example $p_{n}=\frac{1}{2}-\frac{\alpha^{2}}{n^{2}}$. In this case we have $4 p_{n} q_{n}$ which converges to one as $\left(p_{\infty}-p_{n}\right)^{2}$.

Theorem 10 We consider a stable non unitary gravitational walk such that for all $n \geq N: p_{n}=\frac{1}{2}$ with a probability decay factor $\rho$ on the absorbing black hole state $s_{0}$. Let t be the remaining black hole lifetime. Let $n \leq N$, when the probability decay factor $\rho$ is smaller than $1-\frac{1}{o(t)}$, the probability sum $r_{n}(t)$ at state $s_{n}$ satisfies the estimate, for $t \rightarrow \infty$ as soon as $n, N=o(\sqrt{n})$.

$$
r_{n}(t)=\rho^{t} F_{n}\left(\frac{1}{\rho}\right)+P\left(t_{n}>t\right)+P\left(t_{n}=t\right)\left(\frac{1}{1-\rho}+\frac{(-1)^{n+t}}{1+\rho}\right)\left(1+O\left(\frac{N}{\sqrt{n+t}}\right)\right),
$$

with

$$
\begin{aligned}
& P\left(t_{n}=t\right)=\sqrt{\pi / 2}\left(\sum_{j=1}^{j=n} D_{j}\right) t^{-\frac{3}{2}}\left(1+O\left(\frac{N}{n+t}\right)\right) \\
& P\left(t_{n}>t\right)=\sqrt{2 \pi}\left(\sum_{j=1}^{j=n} D_{j}\right) t^{-\frac{1}{2}}\left(1+O\left(\frac{N}{n+t}\right)\right) .
\end{aligned}
$$

Proof: The analysis of the stable gravitational walk and the decomposition $F_{n}(u)=\prod_{j=1}^{j=n} G_{j}(u)$ and $K_{n}(u)=2 p_{n} u G_{n}(u)=H_{n}(u)-\sqrt{s(u)} Q_{n}(u)$ remains with the asymptotic estimates. Nevertheless the contribution of $g_{\rho}(\bar{u})$ becomes singular when $\bar{u}=1$. In this case we have to develop the asymptotics of $P\left(t_{n}>t\right)$ separately and this become the preponderant term. It comes that $r_{n}(t)=\rho^{t}+P\left(t_{n}>\right.$ $t)+P\left(t_{n}=t\right)\left(\frac{1}{1-\rho}+\frac{(-1)^{n+t}}{1+\rho}\right)\left(1+O\left(\frac{N}{\sqrt{n+t}}\right)\right)$ with

$$
\begin{aligned}
& P\left(t_{n}=t\right)=\sqrt{\pi / 2}\left(\sum_{j=1}^{j=n} D_{j}\right) t^{-\frac{3}{2}}\left(1+O\left(\frac{N}{n+t}\right)\right) \\
& P\left(t_{n}>t\right)=\sqrt{2 \pi}\left(\sum_{j=1}^{j=n} D_{j}\right) t^{-\frac{1}{2}}\left(1+O\left(\frac{N}{n+t}\right)\right) .
\end{aligned}
$$

And we have

$$
D_{n}=\frac{p_{n}}{q_{n}} D_{n+1}=\prod_{j=n}^{j=\infty} \frac{p_{j}}{q_{j}}
$$

Notice that:

$$
D_{n}=\exp \left(2\left(V_{n}-V_{\infty}\right)\right) .
$$

The transition to generalized gravitational walks is somewhat different because $4 p_{n} q_{n}$ converges to one like $\left(p_{\infty}-p_{n}\right)^{2}$ since $p_{\infty}=\frac{1}{2}$ instead of converging to $4 p_{\infty} q_{\infty}$ like $p_{\infty}-p_{n}$ when $p_{\infty}<\frac{1}{2}$. Therefore the condition of the transition to the asymptotics of the previous section is now that the series in $p_{\infty}-p_{n}$ converges (instead of the series in $\sqrt{p_{\infty}-p_{n}}$ ).

We now consider the case where $\rho$ is very close to 1 .

Corollary 4 We consider a stable non unitary gravitational walk such that for all $n \geq N: p_{n}=\frac{1}{2}$ with a probability decay factor $\rho$ on the absorbing black hole state $s_{0}$. Let $t$ be the remaining black hole lifetime. Let $n \leq N$, when the probability decay factor $\rho$ belongs to an interval of the kind $] 1-o\left(\frac{1}{\sqrt{t}}\right), 1-\frac{1}{o(t)}[$ 


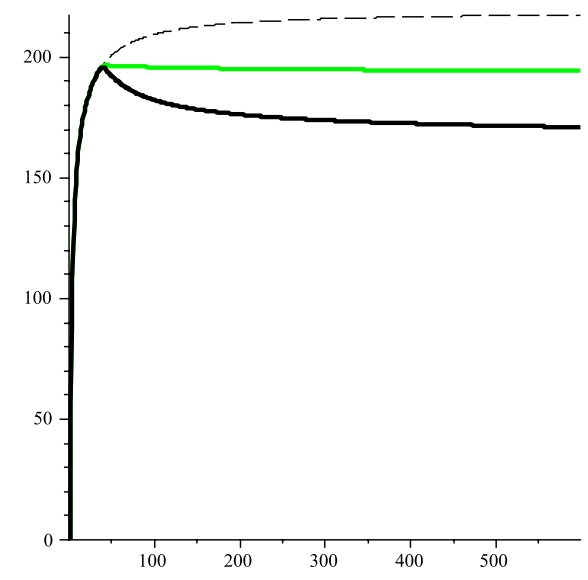

(a) $\rho=\exp \left(-50.10^{-8}\right)$

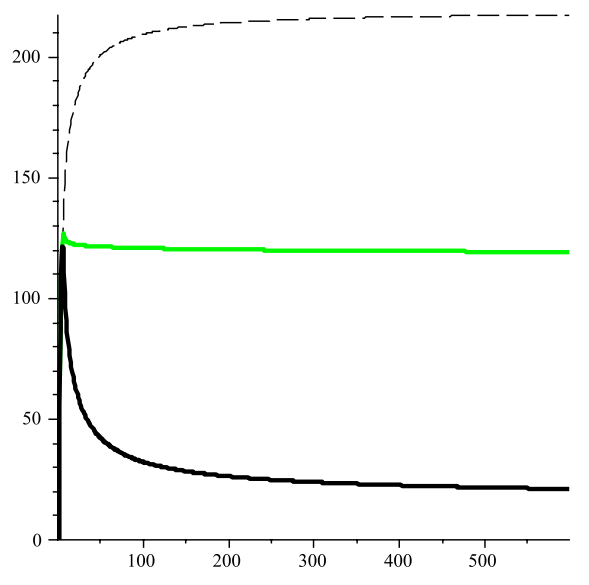

(b) $\rho=\exp \left(-200.10^{-8}\right)$

Fig. 11: Actual potential (dashed) and apparent asymptotic potential (plain) for a gravitational walk with $p_{n}=$ $\frac{1}{2} \exp \left(-\frac{1000}{n^{2}}\right)$ and $t=10^{8}$. In green we display the average value between actual and apparent potential.

the probability sum $r_{n}(t)$ at state $s_{n}$ for the black hole lifetime $t$ satisfies the estimate, for $t \rightarrow \infty$ as soon as $n, N=o(\sqrt{n})$ :

$$
r_{n}(t)=\rho^{t}+P\left(t_{n}>t\right)(1+o(1)) .
$$

Proof: For this model we get $\frac{1}{1-\rho} P\left(t_{n}=t\right)=o\left(P\left(t_{n}>t\right)\right)$ and since $F_{n}(1)=1$ we get the required estimate.

In other words when $\rho^{t}=o(1)$, the non unitary effect is equivalent to end pay back model: if the rabbit reaches the black hole at any time before time $t$, the unitary effect is $\rho^{t}$, otherwise it is 1 . Indeed we should have $r_{n}(t)=\rho^{t}+P\left(t_{n}>t\right)\left(1-\rho^{t}\right)=\rho^{t}+P\left(t_{n}>t\right)(1+o(t))$.

The quasi continuous condition brings a similar conclusion as in the previous section. Defining

$$
\int_{z}^{\infty} \log \left(\frac{p(x)}{q(x)}\right) d x=\alpha \log \left(\rho^{t} t^{\frac{1}{2}}\right) .
$$

The black hole is attractive until state $s_{B}$ such that $B=\left\lfloor\frac{z}{\alpha}\right\rfloor$ with $\tilde{p}_{n}(t) \approx p_{n}$ : the attraction is unchanged. Beyond state $s_{B}$ the random walk is repulsive with $\tilde{p}_{n} \approx q_{n}:$. the attraction is completely reversed beyond that state. See Figure 11

In quasi continuous situation, we denote $V(y)=\int_{0}^{y} \frac{1}{2} \log \left(\frac{q(x)}{p(x)}\right) d x$ and $\Delta V(y)=V(\infty)-V(y)$. For $x=\alpha n$ it turns out that $V_{n}=\frac{1}{\alpha} V(x)+O(1)$ and $D_{n}=\exp \left(-\frac{2}{\alpha}(V(x)-V(\infty))\right.$. Similarly $\sum_{j=1}^{j=n} D_{j}=\frac{\exp \left(\frac{2}{\alpha}(V(x)-V(\infty))\right.}{1-\frac{p(x)}{q(x)}}$. Denoting $\rho=\exp \left(-\frac{\beta}{t}\right)$, we have

$$
r_{n}=e^{-\beta}+\frac{\sqrt{2 \pi}}{\sqrt{t}} \frac{\exp \left(-\frac{2}{\alpha} \Delta V(x)\right)}{1-\frac{p(x)}{q(x)}}\left(1+\frac{1}{2 \beta}+O\left(\frac{1}{t}\right)\right) .
$$




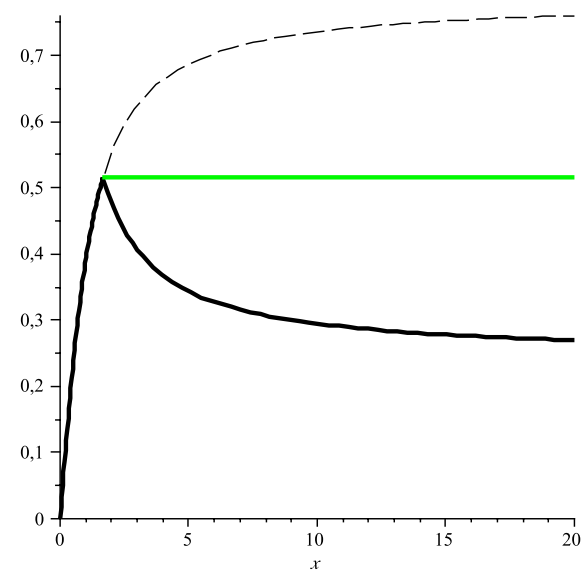

Fig. 12: Actual potential $V(x)$ (dashed) and apparent asymptotic potential $\tilde{V}(x)$ (plain) for a gravitational walk with $p_{n}=1-\frac{1}{1+\exp \left(-\frac{1}{1+x^{2}}\right)}, t=10^{90}, \beta=10^{20}, \alpha=\frac{1}{2} \cdot 10^{-20}$, in green we display the average value between actual and apparent potential.

Assuming $t$ and $\frac{1}{\alpha}$ large, the change of mode occurs for $z$ such that

$$
\beta=\frac{1}{2} \log t+\frac{2}{\alpha} \Delta V(z)+\log \left(1-\frac{p(z)}{q(z)}\right) .
$$

We can investigate a special case where the computations are tractable. For example when $\log \left(\frac{q(x)}{p(x)}\right)_{\tilde{\sim}}=$ $\frac{1}{1+x^{2}}$, namely $p_{n}=1-\frac{1}{1+\exp \left(-\frac{1}{1+x^{2}}\right)}$. In this case we have $V(x)=\frac{1}{2} \arctan (x)$. Denoting $\tilde{V}(x) \stackrel{p(x)}{=} \alpha \tilde{V}_{n}$, Figure 12 displays the actual and apparent potential for this case with parameters tuned to galactic orders of magnitudes: $t=10^{90}, \alpha=\frac{1}{2} \cdot 10^{-20}, \beta=10^{20}$. We get $z \approx 1.7$.

\section{Physical considerations}

We can apply this result to physically realistic models. Let consider a gas of particles of mass $m$ and at temperature $k$. The probability density $P(v)$ that (the radial component of) the speed is equal to $v$ follows Boltzmann density:

$$
P(v)=\sqrt{\frac{m}{2 k T \pi}} \exp \left(-\frac{m v^{2}}{2 k T}\right)
$$

where $k$ is the Boltzmann constant: $k \approx 1.3806503 \times 10^{-23} \mathrm{~m}^{2} \mathrm{~kg} \mathrm{~s}^{-2} \mathrm{~K}^{-1}$. The attraction of a body of mass $M_{S}$ at distance $r$ is given by the gravitational acceleration $g(r)=\frac{M_{S} G}{r^{2}}$, where $G \approx 6.67300 \times$ $10^{-11} \mathrm{~m}^{3} \mathrm{~kg}^{-1} \mathrm{~s}^{-2}$ is the gravitational constant.

Assume that the particle has a collision every $\theta$ seconds, and at each collision it takes a new speed $v$ according to Boltzmann density without memory, like a pure quantum event. Simplifying our model to assume the particle switch to state $s_{n}$ to state $s_{n+1}$ when $-g(r) \frac{\theta^{2}}{2}+v \theta>0$ we get

$$
p(r)=\frac{1}{2}\left(1-\operatorname{erf}\left(\sqrt{\frac{m}{2 k T}} \frac{\theta g}{2}\right)\right),
$$


with $\operatorname{erf}(x)$ the error function: $\operatorname{erf}(x)=\int_{0}^{x} \frac{2}{\sqrt{\pi}} \exp \left(-y^{2}\right) d y$. When $r$ is large we have

$$
p(r) \approx \frac{1}{2}\left(1-\sqrt{\frac{m}{2 k T \pi}} \frac{M_{S} G \theta}{r^{2}}\right),
$$

and

$$
\Delta V(r) \approx \sqrt{\frac{m}{2 k T \pi}} \frac{M_{S} G}{r} \theta=\sqrt{\frac{m}{2 k T \pi}} \Delta V_{G}(r) \theta
$$

where $\Delta V_{G}(r)$ is the gravitational potential of body $M_{S}$ at distance $r$. Remains to express $\alpha$ as a function of $\theta$ and $m$ and $T$. In our model $\alpha$ is the average free (radial) distance travelled by a particle between two collisions. If the average time is $\theta$ then $\alpha=E(|v|) \theta$.

Since $E(|v|)=\sqrt{\frac{2 k T}{m \pi}}$ we get

$$
r_{n}=e^{-\beta}+\frac{\sqrt{2 \pi}}{\sqrt{t}} \frac{\exp \left(-\frac{m}{k T} \Delta V_{G}(x)\right)}{1-\frac{p(x)}{q(x)}}\left(1+\frac{1}{2 \beta}+O\left(\frac{1}{t}\right)\right) .
$$

The change of mode occurs around $z$ such that (neglecting $\frac{1}{t}, \frac{1}{\beta}$ and log terms)

$$
\beta=\frac{m}{k T} \Delta V_{G}(z)=\frac{m G M_{S}}{k T z} .
$$

It is worthy to notice that critical $z$ does not depend on inter-collision average period. In other words, it is independent of particle density and particle critical section, it only depends on temperature and individual mass. For an evaluation of the order of magnitude we have $M_{S} \approx 10^{42}$ (including dark matter). Taking $m=10^{-27} \mathrm{~kg}, T=10,000 \mathrm{~K}$ we get with rule of the thumb estimate $z \approx 10^{21}: \beta \approx 10^{3}$.

If we denote $v_{L}(r)$ the liberation speed that is needed to leave the galaxy from distance $r$ we have

$$
\frac{v_{L}^{2}}{2}=\Delta V_{G}(r)
$$

and therefore $e^{-\beta}=\exp \left(-\frac{m v_{L}^{2}}{2 k T}\right)$, in other words the apparent mode transition occurs when the quantity $\rho^{t}=e^{-\beta}$ is of the order of the probability of liberation speed.

The bimodal aspect of the apparent gravitational walk, and in particular the exact reversion of the random walk beyond critical distance $z$ give some reminiscence about the mysterious dark energy effect. Since 1998, it has been noted that the expansion of the universe is in acceleration, fact that contradicts the usual decelerated expansion scheme predicted by general relativity. In order to explain this discrepancy, astrophysicists have imagined the introduction of a so-called dark energy that contributes to the acceleration of the expansion. The reversion of gravitational potential, in the gravitational walk could be a candidate effect that contributes to dark energy, assuming that galactic black holes are non unitary. The impact on Friedmann-Lemaitre equations must be investigated.

It should be noted that in the model presented in Figure 13 we would have a non unitary effect of less than $10^{-70}$ per time unit, inside a black hole. It means that the impact of the non unitary effect within the critical distance is not measurable, and that only the extremely large black hole lifetimes would make it apparent beyond the critical distance. It should also be noted that the repulsion is not due to a force in the 


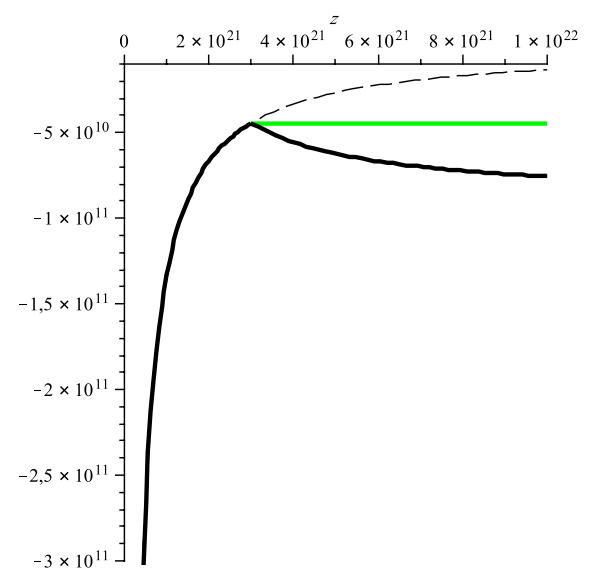

Fig. 13: Actual potential $-\Delta V_{G}(x)$ (dashed) and apparent asymptotic potential $\Delta \tilde{V}_{G}(x)$ (plain) for a gravitational walk with realistic setting $M_{S}=2 \times 10^{42} \mathrm{~kg}$ ( 1 trillion solar masses), critical $z=3 \times 10^{21} \mathrm{~m}$ (300,000 light-years), in green we display the average value between actual and apparent potential.

physical sense, but would be due to a weird effect in future trajectory weight summation in the random walk.

The identity $\beta=\frac{m G M_{S}}{k T z}$ reminds the formula for the incremental entropy carried by a mass $m$ in a black hole of mass $M_{B}: S_{B}=8 \pi \frac{m G M_{B}}{c \hbar}$. This suggests that the ratio $\frac{\beta}{S_{B}}$ somewhat measures the ratio of information lost by the black hole during its evaporation. Assuming $M_{B}=M_{S}$, it is interesting to notice that this ratio, equal to $\frac{\hbar c}{8 \pi k T z}$, does not depend on the gravitational constant $G$ and on the mass of the black hole, it turns out that it would be of the order $10^{-29}$.

\section{Conclusion and perspective}

We have analyzed the effect of a non unitary black hole state in a discrete random walk model. Even simplistic this model would be very difficult to simulate since it would require of the order of $10^{90}$ steps over $10^{20}$ states. We have proven that in the most simplistic scenarios the non unitary effect is very sensitive to the tuning of the parameters, but in most case lead to a reversion of the random walk potential beyond a certain range. This effect has interesting interpretation in physics that would probably need to be investigated in a separate track.

The analysis tools are continued fractions, complex analysis and singularity analysis. In passing we get interesting insights about the time distribution of return times in concave random walks. There are of course many technical points that need further investigation. What is the correct singularity analysis when the series $\sqrt{p_{\infty}-p_{n}}$ diverges (or $p_{\infty}-p_{n}$ diverges in case of gravitational walk)? It seems that in this case we have $K_{n}(u)=H_{n}(u)-(\bar{u}-u)^{a_{n}} Q_{n}(u)$ where $a_{n} \rightarrow \frac{1}{2}$ when $n \rightarrow \infty$.

An extension to continuous time and state space would be interesting to analyze, very likely via partial derived equations. The result of the reversion of the potential would be interesting to investigate in multidimensional non unitary random walks (see Figure 14). 


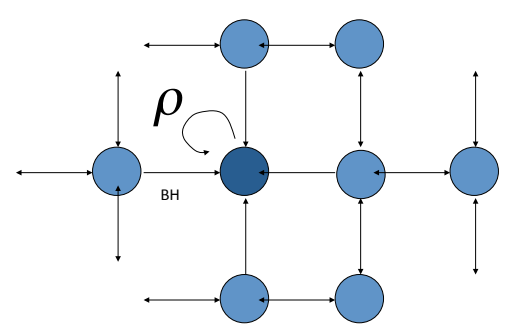

Philippe Jacquet

Fig. 14: Two dimensional random walk.

\section{Acknowledgements}

The author wants to thanks Philippe Flajolet for his immense competence, his humanity, his continual support, and his illuminating remarks, in particular when he mentioned the continued fractions methodology when a preliminary version of this work was presented during the workshop in honor of his 60th birthday.

\section{References}

[1] P. Flajolet, "Combinatorial Aspects of Continued Fractions", in Discrete Mathematics 32, pp. 125-161, 1980.

[2] S. Hawking, "Breakdown of Predictability in Gravitational Collapse", Physical Review D 14: 24602473, 1976.

[3] P. Flajolet, A. Odlyzko , "Singularity analysis of generating functions”, SIAM J. Discrete Math., 1990.

[4] P. Kirschenhofer, H. Prodinger, "The higher moments of the number of returns of a simple random walk", Advances in Applied Probability, 1994.

[5] J. Traschen, "An Introduction to Black Hole Evaporation", arXiv:gr-qc/0010055v1, Published in Mathematical Methods of Physics, proceedings of the 1999 Londrina Winter School, editors A. Bytsenko and F. Williams, World Scientific, 2000.

[6] A. Ambainis, E. Bach, A. Nayak, A. Vishwanath, J. Watrous, "One-dimensional quantum walks", Proceedings of the thirty-third annual ACM symposium on Theory of computing, 2001.

[7] C. Banderier, P. Flajolet, "Basic analytic combinatorics of directed lattice paths", Theoretical Computer Science, vol. 281 Issues 1-2, 3, Pages 37-80, 2002.

[8] S. De Filippo, F. Maimone, A. Robustelli, "Numerical simulation of nonunitary gravity-induced localization", Physica A330, 459-468, 2003.

[9] E. Bender, G. Lawler, R. Pemantle, H. Wilf, "Irreducible compositions and the first return to the origin of a random walk", Séminaire Lotharingien de Combinatoire, 2004.

[10] V. Springel, S. White, A. Jenkins, C. Frenk, N. Yoshida, L. Gao, J. Navarro, R. Thacker, D. Croton, J. Helly, J. Peacock, S. Cole, P. Thomas, H. Couchman, A. Evrard, J. Colberg, F. Pearce, " Simulations of the formation, evolution and clustering of galaxies and quasars," Nature, 2005.

[11] S. Hawking, "Information Loss in Black Holes", arXiv:hep-th/0507171, Physical Review D 72: 084013, 2005.

[12] A. Bressler , R. Pemantle, "Quantum random walks in one dimension via generating functions," DMTCS Proceedings, 2007 Conference on Analysis of Algorithms, AofA 07, 2007. 